\title{
NUEVOS RESTOS DE CIERVOS (CERVIDAE, MAMMALIA) PARA EL PLEISTOCENO DE LA REGIÓN PAMPEANA (ARGENTINA): CONSIDERACIONES PALEOBIOGEOGRÁFICAS Y PALEOECOLÓGICAS
}

\author{
NICOLÁS ROBERTO CHIMENTO \\ Laboratorio de Anatomía Comparada y Evolución de los Vertebrados, Museo Argentino de Ciencias Naturales "Bernardino \\ Rivadavia", Av. Ángel Gallardo 470 (C1405DJR), 4982-6595 int.162, Buenos Aires, Argentina. \\ nicochimento@hotmail.com \\ JOSÉ IGNACIO ZUCCARI, JOSÉ MARÍA MARCHETTO \\ Museo Legado del Salado, Almafuerte 308 (CP 6000), Junín, Buenos Aires, Argentina. \\ ignaciozuccari@hotmail.com,jose_marche@hotmail.com \\ LORENA BERBACH \\ Museo Municipal de Ciencias Naturales “Carlos Darwin”, Urquiza 123 (B8109FWC), Punta Alta, Buenos Aires, Argentina. \\ lberbach@yahoo.com
}

\begin{abstract}
New remains of deers (Cervidae, Mammalia) of the Pleistocene from the Pampean Region (Argentina): paleobiogeographic and paleoecological considerations. Cervidae are an Cetartiodactyla family that entered South America in the Pleistocene, during the event known as "Great American Biotic Exchange". In the Argentinean Quaternary the genera Antifer, Epieuryceros, Morenelaphus and Paraceros, all extinct in the Late Pleistocene, are recognized along with the genera Hippocamelus, Pudu, Blastoceros, Ozotoceros and Mazama, present inhabitants of the South American territory, and some of which are represented also in Pleistocene times. The aim of this work is to present and describe new fossil deer remains from different Pleistocene localities in Buenos Aires Province (Argentina) and to make some geographical and environmental considerations. The southernmost record of the genus Morenelaphus and the species M. lujanensis is here presented, coming from Punta Alta locality, and the first female specimen assigned to Morenelaphus sp. is described, as well as new materials with the same assignment for the localities of Junín and Paraje 7 Bridges, Sauce Grande River Valley. New remains of Antifer sp., from San Bernardo locality, and Hippocamelus sulcatus from Junín locality are disclosed. The first Pleistocene record of Ozotoceros bezoarticus is presented for the Buenos Aires Province, coming from the Junín locality.
\end{abstract}

Keywords. Morenelaphus, female, Antifer, Hippocamelus, Ozotoceros, replacement.

RESUMO - Os Cervidae constituem uma das famílias de Cetartiodactyla que ingressaram na América do Sul no Pleistoceno, como parte do evento conhecido como "Grande Intercâmbio Biótico Americano". No Quaternário argentino são reconhecidos os gêneros Antifer, Epieuryceros, Morenelaphus e Paraceros, extintos no Neopleistoceno, além dos gêneros Hippocamelus, Pudu, Blastoceros, Ozotoceros e Mazama, habitantes atuais do território sul-americano, alguns dos quais se encontram representados também no Pleistoceno. O presente estudo tem por objetivo dar a conhecer e descrever novos materiais de cervos fósseis procedentes de distintas localidades pleistocênicas da Província de Buenos Aires, Argentina, bem como discutir sobre a distribuição geográfica dos mesmos. É apresentado o registro mais austral do gênero Morenelaphus e da espécie M. lujanensis, correspondente a localidade de Punta Alta, e descrito o primeiro exemplar fêmea assignado a Morenelaphus sp., além de novos materiais com a mesma assignação para as localidades de Junín e Paraje 7 Puentes, Vale do Rio Sauce Grande. É dado a conhecer novos materiais de Antifer sp. para a localidade de San Bernardo, e Hippocamelus sulcatus para a localidade de Junín. É também apresentado o primeiro registro pleistocênico de Ozotoceros bezoarticus para a Província de Buenos Aires, procedente da localidade de Junín.

Palavras-chave: Morenelaphus, fêmea, Antifer, Hippocamelus, Ozotoceros, substituição. 


\section{INTRODUCCIÓN}

Los Cervidae constituyen una de las familias de Cetartiodactyla que ingresó a América del Sur en tiempos pleistocénicos, durante el evento conocido como "Gran Intercambio Biótico Americano" (Webb, 2000; Cione et al., 2015). En este continente tuvieron una amplia diversificación, lo cual ha llevado a reconocer varios géneros y especies, muchas de las cuales habitan actualmente el territorio argentino (ver Carette, 1922; Hershkovitz, 1982). El conocimiento de los ciervos fósiles se ha basado mayormente en la morfología de las cornamentas, estructuras que poseen una forma y tamaño variable respecto al desarrollo ontogenético y otros aspectos intraespecíficos (Carette, 1922; Cabrera, 1929, 1943; Azorit et al., 2002). Esto ha llevado a reconocer numerosos representantes extintos de esta familia, ya que los restos de astas son bastante frecuentes en los depósitos cuaternarios de Argentina fundamentalmente, y el resto de Sudamérica. Sin embargo, revisiones detalladas recientes han dado cierta luz en la composición taxonómica de sus representantes fósiles (Menegaz, 2000; Alcaraz, 2010). En efecto, en el Cuaternario argentino se reconocen los géneros Antifer (Ameghino, 1889), Epieuryceros (Ameghino, 1889), Morenelaphus (Carette, 1922) y Paraceros (Ameghino, 1889), extinguidos en el Pleistoceno Tardío, además de los géneros Hippocamelus (Leuckart, 1816), Pudu (Gray, 1852), Blastoceros (Gray, 1872), Ozotoceros (Ameghino, 1891) y Mazama (Rafinesque, 1817), habitantes actuales del territorio sudamericano y algunos de los cuales se encuentran representados también en tiempos pleistocénicos [e.g. Hippocamelus, Mazama, Ozotoceros (Ameghino 1888, 1889, 1902; Kraglievich, 1932; Vezzosi, 2010; Alcaraz \& Francia, 2013)]. El objetivo de este trabajo es dar a conocer y describir nuevos materiales de ciervos fósiles procedentes de distintas localidades pleistocénicas de la Provincia de Buenos Aires, Argentina, y discutir sobre la distribución geográfica de los mismos.

\section{MATERIAL Y MÉTODOS}

Los materiales estudiados fueran colectados en distintas localidades pleistocénicas de la Provincia de Buenos Aires: Junín, San Bernardo, Paraje 7 Puentes y Playa del Barco (Figura 1).

Se sigue el esquema cronoestratigráfico propuesto por Cione et al. (2015, y bibliografía allí citada). Adicionalmente, se utiliza la escala temporal propuesta según la International Chronostratigraphic Chart (2018). La nomenclatura de las estructuras anatómicas que forman las astas está tomada de Loponte (2005; ver también Alcaraz, 2010; Labarca \& Alcaraz; 2011), mientras que la nomenclatura oclusal dentaria fue tomada de Pêgo (2014). Los materiales estudiados se compararon con especímenes de cérvidos actuales y fósiles depositados en colecciones oficiales.

Abreviaturas institucionales: MACN-Pv, Sección Paleontología Vertebrados, Museo Argentino de Ciencias Naturales "Bernardino Rivadavia", Ciudad Autónoma de Buenos Aires, Argentina; MD-PDB, Colección Playa del
Barco, Museo Municipal de Ciencias Naturales "Carlos Darwin”, Punta Alta, Buenos Aires, Argentina; MHJ, Museo Histórico de Junín, Buenos Aires, Argentina; MLP, División Paleontología Vertebrados, Museo de La Plata, Buenos Aires, Argentina; MLS, Museo Legado del Salado, Junín, Buenos Aires, Argentina; MPA, Colección Bianco, Museo Municipal de Ciencias Naturales "Carlos Darwin", Punta Alta, Buenos Aires, Argentina; MPB, Museo Paleontológico Bariloche, Bariloche, Argentina; PV.UNS, Paleontología Vertebrados, Universidad Nacional del Sur, Bahía Blanca, Buenos Aires, Argentina.

\section{PALEONTOLOGÍA SISTEMÁTICA}

\author{
Clase MAMMALIA \\ Superorden CETARTIODACTYLA \\ Montgelard et al., 1997 \\ Suborden RUMINANTIA Scopoli, 1777 \\ Superfamilia CERVOIDEA Simpson, 1931 \\ Familia CERVIDAE Gray, 1821
}

Morenelaphus Carette, 1922

Morenelaphus sp.

(Figuras 2-5)

Material referido. MPA-85-189-I-D, parte posterior de cráneo con asta derecha fragmentaria y sólo parte del pedúnculo izquierdo. Se conserva la región occipital y el basicráneo casi completos (Figuras 2A-C); MPA-85-251-I-D, fragmento distal de asta con el tercer candil completo y parte de la bifurcación del cuarto candil y la corona (Figura 2E); PV.UNS 4000 , dos fragmentos de astas (Figuras $2 \mathrm{~F}-\mathrm{G}$ ); PV.UNS 4003, fragmento proximal de asta (Figura 2H); PV.UNS 4004, fragmento proximal de asta (Figura 2I); PV.UNS 4036, fragmento proximal de asta con parte de la roseta y la garceta casi completa (Figura 2J); MHJ 0538, fragmento proximal de asta derecha con la roseta, el pedúnculo, la parte basal de la garceta y parte proximal de la vara antes de la salida del segundo candil (Figura 2D); MD-PDB-05-110, parte posterior de cráneo de un ejemplar hembra (sin astas) conservando parte del basicráneo (Figura 3) y un pequeño fragmento de maxilar derecho con el M3 completo (Figura 4); MPA-845-I-D, hemimandíbula izquierda fragmentada (Figura 5C); MPA-85-4-I-D, hemimandíbula derecha casi completa (Figura 5D); MD-PDB-05-02, fragmento de hemimandíbula derecha con dos premolares y tres molares completos (Figura 5A); MD-PDB-05-108, fragmento de hemimandíbula con parte de un molar (Figura 5B).

Procedencia geográfica y estratigráfica. Los materiales MPA-85-189-I-D (Figuras 2 A-C), MD-09-01, MPA-85251-I-D, MD-PDB-05-110, MPA-84-5-I-D, MPA-85-4-I-D provienen de la localidad Playa del Barco (6134'52"O y 3900'09'S), partido de Coronel Rosales, Provincia de Buenos Aires. Fueron hallados en los niveles conglomerádicos asignados al Pleistoceno Tardío (Vega et al., 1989; Aramayo 

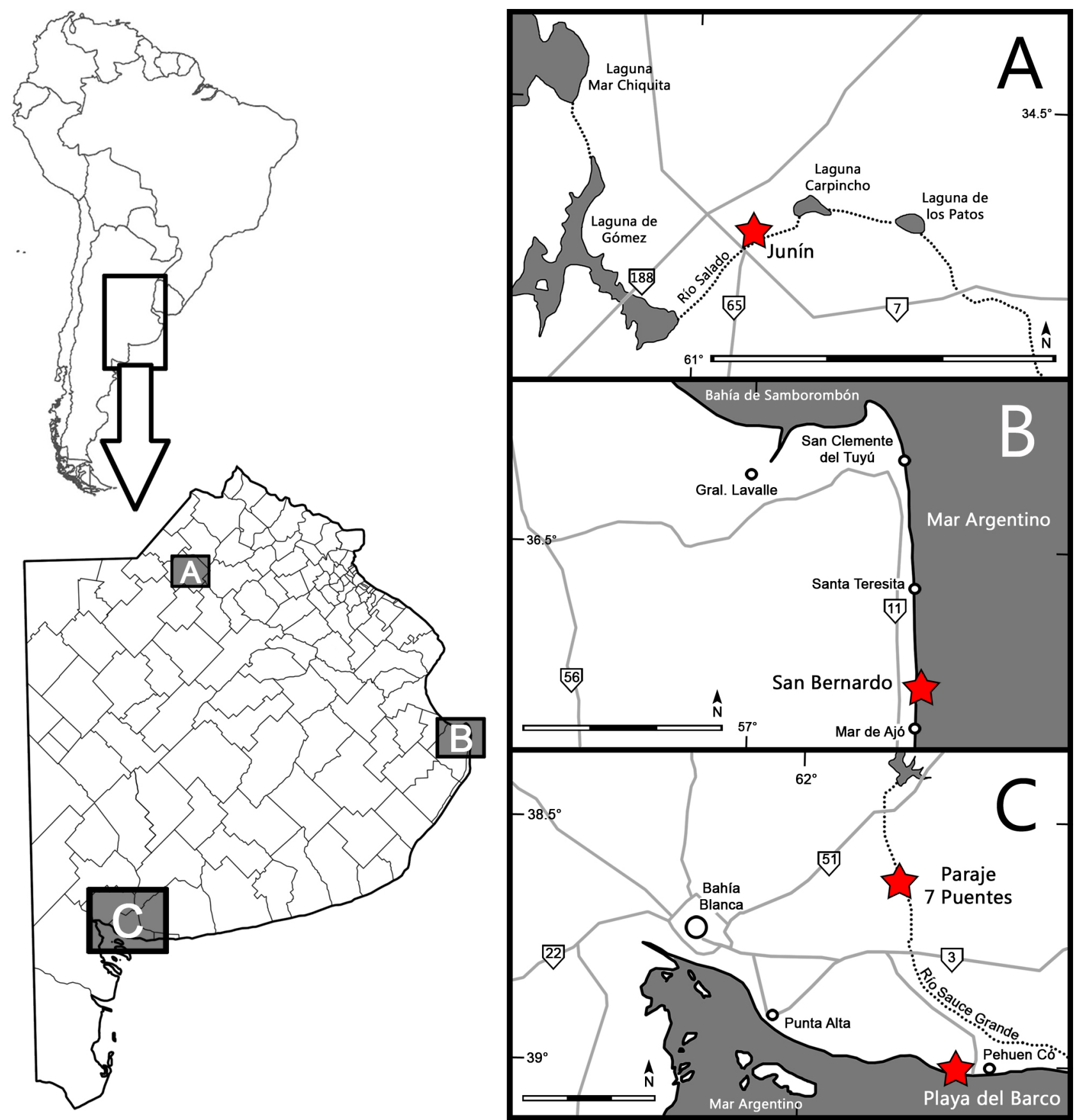

Figura 1. Ubicación de las áreas donde fueron hallados los ejemplares. A, detalle de la zona norte de la Provincia de Buenos Aires, donde está la localidad de Junín; B, detalle de la costa este de la Provincia de Buenos Aires, donde está la localidad de San Bernardo; C, detalle de la costa sur de la Provincia de Buenos Aires, donde están las localidades de Paraje 7 Puentes y Playa del Barco. Las estrellas muestran las localidades donde se colectaron los nuevos restos descritos. Escalas $=30 \mathrm{~km}$.

Figure 1. Map showing the areas where the specimens were found. A, detail of the northern area of Buenos Aires Province, where the Junin locality is placed; B, detail of the east coast of Buenos Aires Province, where the San Bernardo locality is placed; C, detail of the south coast of Buenos Aires Province, where the Paraje 7 Puentes and Playa del Barco localities are placed. Stars showing the localities where the new remains described were found. Scale bars $=30 \mathrm{~km}$.

\& Manera de Bianco, 1989; Zavala \& Quattrocchio, 2001; Aramayo et al., 2002; Tomassini et al., 2010).

Los materiales PV.UNS 4000, PV.UNS 4003 y PV.UNS 4004 (Figuras 2 F-G) fueron hallados en la localidad Paraje 7 Puentes (Figura 1C), en el Valle del Río Sauce Grande, Provincia de Buenos Aires. La localidad Paraje 7 Puentes corresponde al sitio mencionado como Bajo de San José en varias contribuciones (Deschamps \& Borromei, 1992; Deschamps \& Tonni, 1992; Deschamps, 2005). En esta localidad, los materiales fueron colectados en niveles correspondientes a la Secuencia Agua Blanca, asignada al Piso/Edad Lujanense (Pleistoceno Tardío; ver Deschamps, 2005). Por otro lado el ejemplar PV.UNS 4036 fue colectado por Beatriz Gutiérrez Tellez en la barrancas del Río Quequén 
Salado, aguas arriba del sitio "Cascada de Palaeolama". Este material procede de la Unidad D del perfil estratigráfico descrito por Pardiñas et al. (1996) para "Cascada de Palaeolama" y asignada al Piso/Edad Lujanense (Pleistoceno Tardío-Holoceno Temprano, Cione et al., 2015). Para esta localidad y unidad estratigráfica estos autores mencionaron restos mandibulares y postcraneanos de Morenelaphus sp. MHJ 0538 fue hallado en las barrancas del Río Salado, muy cercano a la ciudad de Junín (Figura 1A) en capas de color verdoso, asignadas al Miembro Guerrero de la Formación Luján (Pleistoceno Tardío, ver Tonni et al., 2003).

Descripciones. Los materiales correspondientes a astas son asignados al género Morenelaphus por presentar la siguiente combinación de caracteres: 1) astas dicotómicas que se bifurcan muy cerca de la roseta (Ameghino, 1888); 2) pedúnculo muy corto; 3 ) vara de sección circular aplastada (Carette, 1922); 4) garceta de sección cilíndrica (Alcaraz, 2010) y formando un ángulo casi recto con respecto a la vara (Carette, 1922; Kraglievich, 1932); 5) garceta bifurcada en dos o más puntas; 6) superficie interna con tubérculos bien marcados (Alcaraz, 2010); 7) vara curvada bruscamente en sentido posterolateral después de la garceta. PV.UNS 4000, PV.UNS 4003 y PV.UNS 4004 corresponden a restos muy fragmentarios de astas robustas (ver Figuras 1F y 1I), de sección subcircular, con profundos surcos y tubérculos muy desarrollados.

MD-PDB-05-110 corresponde a la parte posterior de un cráneo (Figura 3), con la parte occipital casi completa, parte del basicráneo, el techo craneano y las paredes laterales parcialmente conservadas. Los frontales no presentan proyecciones ni indicios de ceratóforos para las astas, lo que indica que el ejemplar es una hembra, posiblemente adulta debido a su tamaño y a que las suturas se encuentran bien soldadas. Este cráneo es levemente más grande que Blastoceros dichotomus. Los frontales son anchos y planos. Se observa la presencia del foramen supraorbitario izquierdo, grande y de contorno circular. La sutura frontal-parietal es complicada, de forma interdigitada, con forma de $\mathrm{V}$, donde el vértice de la misma se encuentra en el medio de la articulación del parietal con ambos frontales. El parietal es abovedado, menos ancho que ambos frontales juntos. La parte posterior del parietal está fragmentada. La región occipital se encuentra completa. La superficie occipital presenta un contorno subcircular, muy similar a Ozotoceros, y diferenciándose de los demás géneros actuales, donde existe una inflexión marcada en los bordes laterales. La cresta occipital está sobreelevada en el medio del occipital. Esta cresta es mucho más angosta que en los géneros actuales Blastoceros, Hippocamelus, Pudu, Mazama, y similar a Ozotoceros. A los lados de la cresta occipital, están las fosas occipitales, profundas y anchas, que alcanzan el borde lateral. Estas fosas están mucho menos desarrolladas en los representantes vivientes de cérvidos sudamericanos. Dorsalmente, la región occipital está bien delimitada por una cresta nucal bien marcada. Del lado derecho se conservó parte del proceso paroccipital, el cual presenta forma acuñada, es corto y sobrepasa levemente el nivel ventral del cóndilo occipital. Los cóndilos occipitales son alargados dorsoventralmente, pero robustos, anchos en su parte dorsal, y de contorno subrectangular. El borde medial de ambos cóndilos posee un contorno sigmoidal, terminando ventralmente en un pequeño proceso medial agudo. Los bordes dorsal y lateral de ambos cóndilos son rectilíneos, y el borde ventral, es levemente convexo y se inclina ventromedialmente. Esta morfología de los cóndilos es muy distinta de la de los taxones actuales, en los que los cóndilos son en general mucho más alargados dorsoventralmente y con el borde dorsal bien convexo. El foramen magno es grande, de contorno suboval. En vista ventral se observa que los cóndilos occipitales son anteroposteriormente cortos y anchos, distinto de lo que se observa en los géneros actuales. El borde dorsal del foramen magno alcanza el nivel medio de los cóndilos (en vista ventral), mientras que en los ciervos actuales sudamericanos este borde llega al extremo posterior de los cóndilos. Ambas bulas timpánicas se conservaron casi completas, y muestran un contorno alargado, grácil, poco globosas, en contraposición a lo que se observa en los ciervos sudamericanos actuales, con bulas cortas y muy globosas. El contorno subcircular de todo el occipital, sin la inflexión en el medio de los bordes laterales, la cresta occipital angosta, las fosas occipitales anchas y profundas, los cóndilos occipitales de contorno subrectangular y de bordes dorsales rectilíneos, y las bulas timpánicas alargadas y poco globosas, son rasgos que se encuentran presentes en ejemplares de Morenelaphus con astas (e.g. MACN-Pv 11722, MACNPv 11564, MACN-Pv 117300, MACN-Pv 12082, MLP-Pv 51-VI-11-69, MLP-Pv 63-V-31-1). En efecto, el ejemplar MD-PDB-05-110 es asignado a Morenelaphus sp. Debido a que las especies definidas para el género Morenelaphus (i.e. $M$. brachyceros y $M$. lujanensis) han sido separadas en base a rasgos de la cornamenta, no es posible asignar el ejemplar MD-PDB-05-110 a nivel específico.

Adicionalmente, se conservó un fragmento de maxilar derecho asociado al cráneo, con el M3 completo y una pequeña parte de la raíz anterior del arco cigomático (Figura 4). El M3 no presenta un desgaste elevado, dado que las cúspides principales (protocono, hipocono, metacono y paracono) no son superficies planas y amplias labiolingualmente, sino que se presentan bien diferenciadas como cúspides redondeadas. En contraposición la fosa del trigón y la fosa del talón son grandes. Otro rasgo indicador de poco desgaste en Morenelaphus, es la presencia de un endostilo bien diferenciado, sin fusionarse entre el protocono y el hipocono (Pêgo, 2014). El M3 está formado por dos lóbulos, donde el lóbulo anterior es más ancho labiolingualmente que el lóbulo posterior. En el lóbulo anterior hay una cresta lingual uniendo el protocono con el protoconulo, paralela al eje alveolar. El endostilo está separado del resto de la corona y no muestra desgaste. El parastilo, mesostilo y metastilo están bien desarrollados en el borde labial del diente. En el lóbulo posterior, hay una cresta bien marcada y con desgaste a lo largo del borde anterior (entocrista de Hershkovitz, 1982). La fosa del trigón es mucho mayor a la fosa del talón. La morfología oclusal del M3 de MD-PDB-05-110 muestra rasgos que permiten asignar el especimen a Morenelaphus 


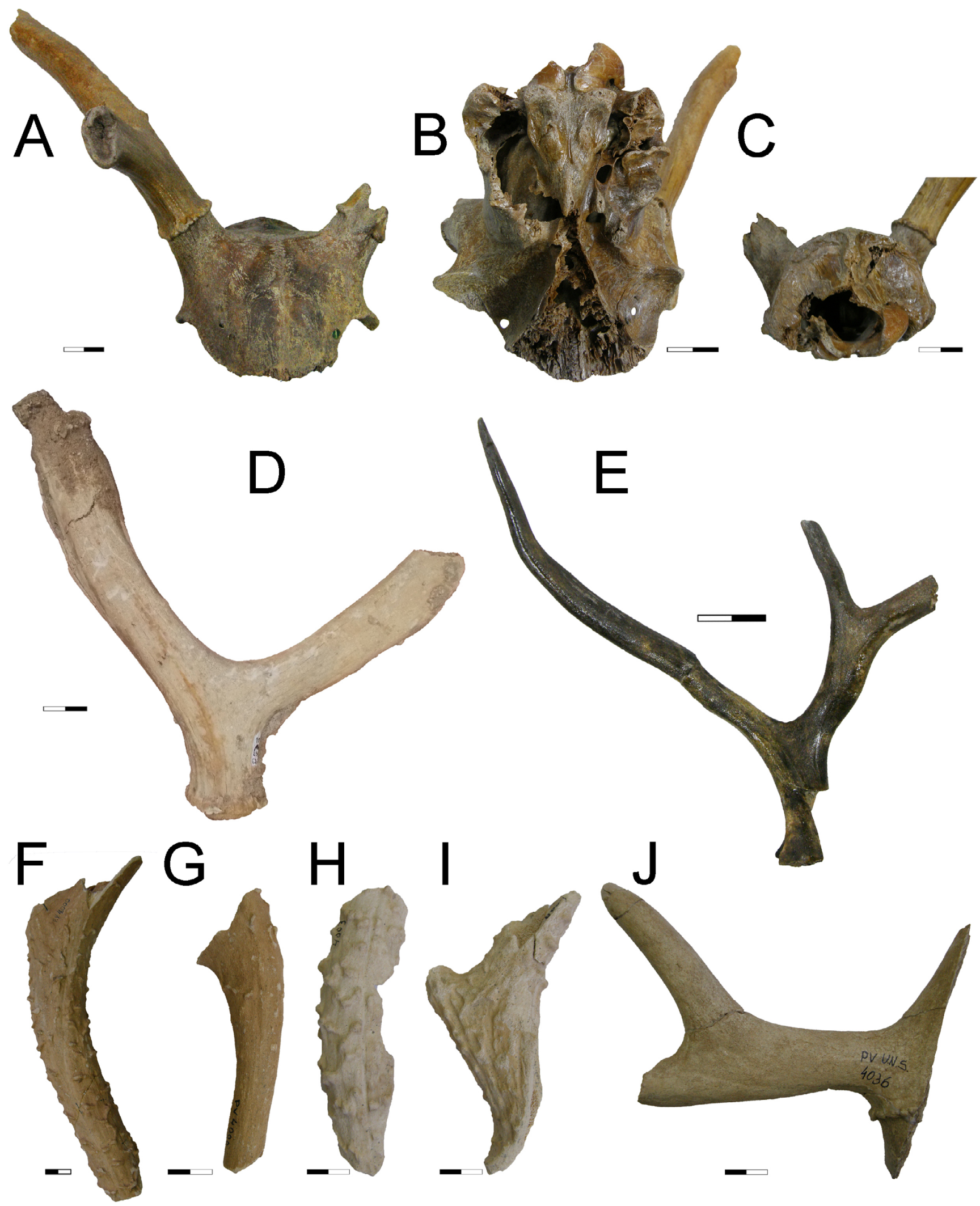

Figura 2. Resto de cráneo con asta y fragmentos de astas asignados a Morenelaphus sp. A-C, MPA-85-189-I-D en vistas frontal (A), ventral (B) y occipital (C); D, MHJ 0538; E, MPA-85-251-I-D; F-G, PV.UNS 4000; H, PV.UNS 4003; I, PV.UNS 4004; J, PV.UNS 4036. Escalas $=20$ mm.

Figure 2. Rest of skull with antler and fragments of antlers assigned to Morenelaphus sp. A-C, MPA-85-189-I-D in frontal (A), ventral (B) and occipital (C) views; D, MHJ 0538; E, MPA-85-251-I-D; F-G, PV.UNS 4000; H, PV.UNS 4003; I, PV.UNS 4004; J, PV.UNS 4036 . Scale bars = 20 mm. 

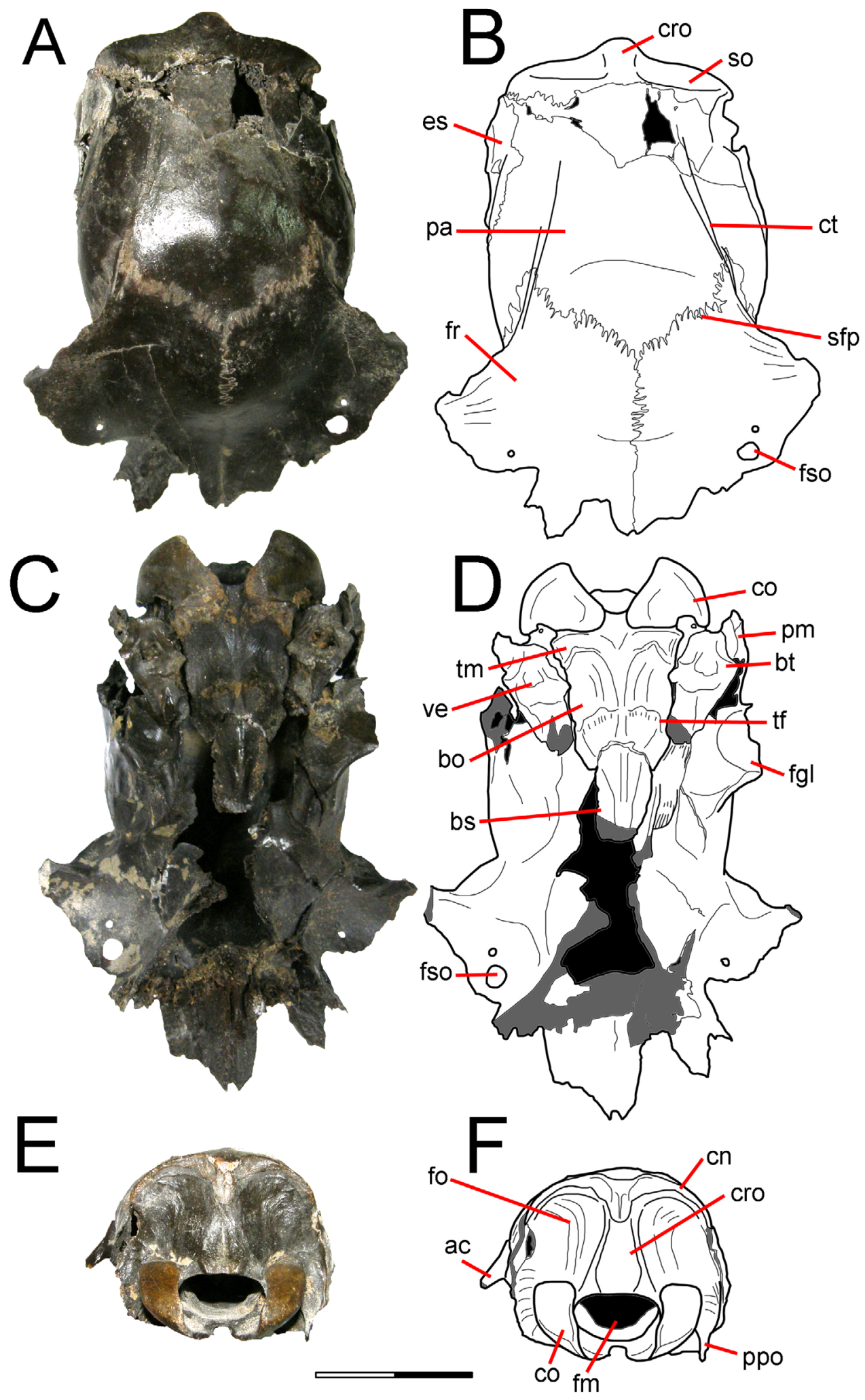

Figura 3. MD-PDB-05-110, cráneo y esquemas en vistas dorsal (A-B), ventral (C-D) y occipital (E-F), asignados a un individuo hembra de Morenelaphus sp. Abreviaturas: ac, arco cigomático; bo, basioccipital; bs, basiesfenoides; bt, bula timpánica; cn, cresta nucal; co, cóndilo occipital; cro, cresta occipital; ct, cresta temporal; es, escamoso; fgl, fosa glenoidea; fm, foramen magno; fr, frontal; fso, foramen supraorbitario; pa, parietal; pm, proceso mastoideo; ppo, proceso paroccipital; sfp, sutura fronto-parietal; so, supraoccipital; tf, tubérculo faríngeo; tm, tubérculo muscular; ve, vagina estilohial. Escala $=20 \mathrm{~mm}$.

Figure 3. MD-PDB-05-110, skull and figures in dorsal (A-B), ventral (C-D), and occipital (E-F) views, assigned to a female specimen of Morenelaphus sp. Abbreviations: ac, zigomatic arc; bo, basioccipital; bs, basisphenoides; bt, tympanic bulla; cn, nuchal crest; co, occipital condyle; cro, occipital crest; ct, temporal crest; es, squamosal; fgp, glenoid fossa; fm, magnum foramen; fr, frontal; fso, supraorbital foramen; pa, parietal; pm, mastoid process; ppo, paroccipital process; sfp, fronto-parietal suture; so, supraoccipital; tf, faringeal tuber; tm, muscular tuber; ve, stilohyal vagina. Scale bar $=20 \mathrm{~mm}$. 


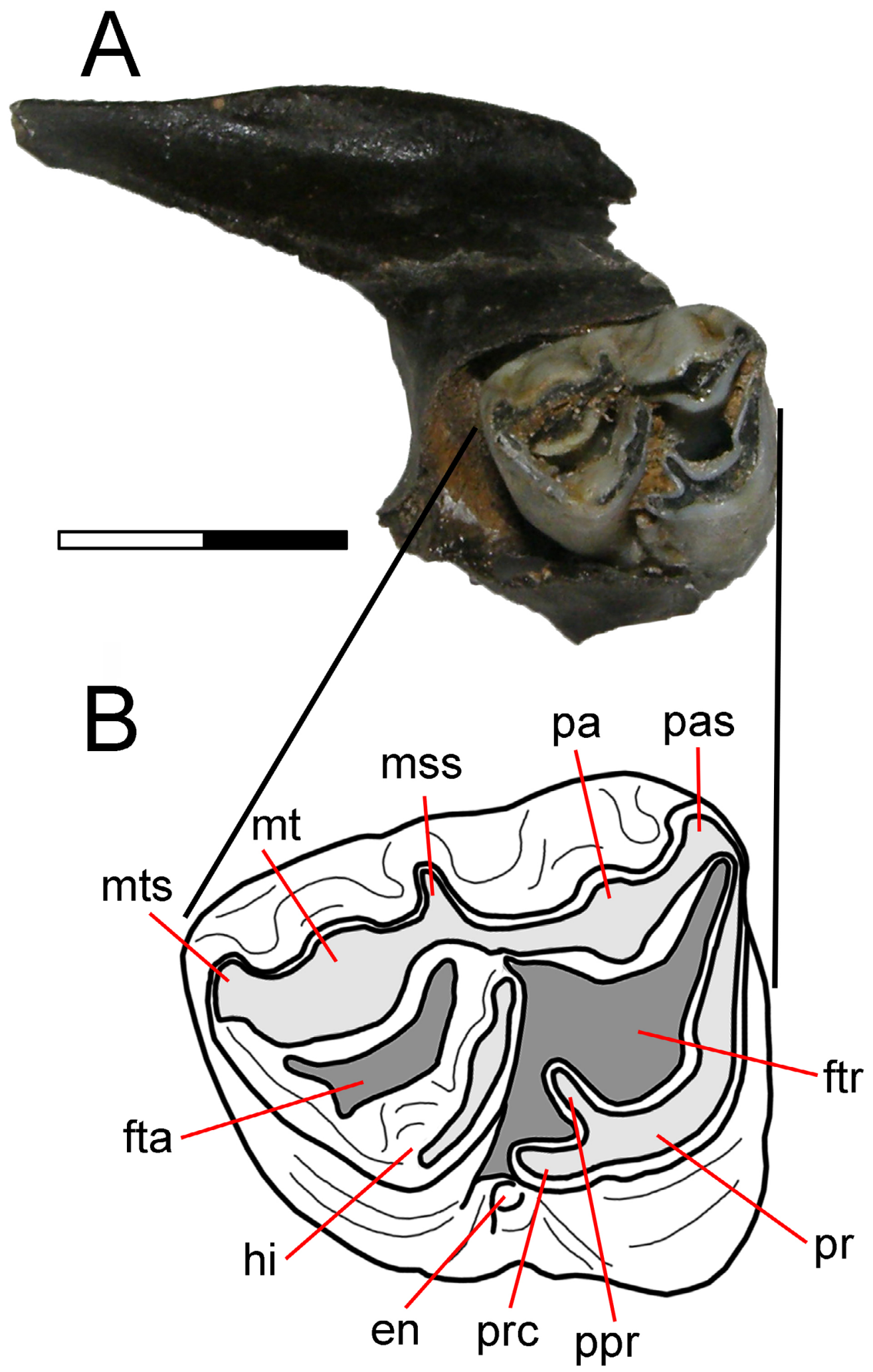

Figura 4. MD-PDB-05-110, fragmento de maxilar con M3, asignados a un individuo hembra de Morenelaphus sp. A, vista oclusal; B, detalle de la morfología dentaria. Abreviaturas: en, endostilo; hi, hipocono; fta, fosa del talón; ftr, fosa do trigón; mt, metacono; mss, mesostilo; mts, metastilo; pa, paracono; pas, parastilo; pr, protocono; pre, protoconulo; ppr, cresta post-protoconal. Escala $=20 \mathrm{~mm}$.

Figure 4. MD-PDB-05-110, maxilar and M3, assigned to a female specimen of Morenelaphus sp. A, occlusal view; B, dental morphology detail. Abbreviations: en, endostyle; hi, hypocone; fta, talon fossa; ftr, trigon fossa; mt, metacone; mss, mesostyle; mts, metastyle; pa, paracone; pas, parastyle; pr, protocone; pre, protoconule; ppr, post-protoconal cresta. Scale bar $=20 \mathrm{~mm}$. 
(Pêgo, 2014), como la fosa del talón en forma de U, con su extremo distal bifurcado con una leve proyección lingual, y la cresta post-protoconal bien definida.

Los materiales MPA-84-5-I-D y MPA-85-4-I-D (Figuras $5 \mathrm{C}-\mathrm{D}$, respectivamente) fueron hallados de forma aislada. Aunque el género Morenelaphus es diagnosticado por características de las astas, varios autores han identificado rasgos en los dientes o en las mandíbulas que lo permiten separar de los taxones actuales. MPA-84-5-I-D y MPA-85-4I-D presentan un diastema mucho más corto que en Blastoceros dichotomus, Ozotoceros bezoarticus, Hippocamelus bisulcus y Odocoileus virginianus, asemejándose a Hippocamelus antisiensis. Una mandíbula robusta y fuerte, con diastema proporcionalmente más corto que en los demás cérvidos sudamericanos, ha sido un rasgo identificado para Morenelaphus por distintos autores (Menegaz, 2000; Alcaraz, 2010; Pêgo, 2014). Adicionalmente, Pêgo (2014) menciona que los bordes labiales de los protocónidos e hipocónidos tienen forma de U en Morenelaphus, mientras que en Blastoceros dichotomus tienen forma de V. Esta forma de U se observa en MPA-84-5-I-D y MPA-85-4-I-D, diferenciándose de $B$. dichotomus y también de los demás cérvidos sudamericanos que muestran forma de $\mathrm{V}$ (e.g. $O$. bezoarticus, $P$. puda, P. mephistophiles, $O$. virginianus, $H$. antisiensis, $H$. bisulcus). En vista lateral, puede verse que MPA-84-5-I-D y MPA-85-4-I-D poseen el borde ventral del diastema fuertemente cóncavo con relación al borde ventral de la rama mandibular a la altura de la serie molar. Este rasgo también se observa en los materiales descritos por Pêgo (2014). En los demás cérvidos sudamericanos, la concavidad es mucho menos pronunciada o incluso casi recta. Por otro lado, el borde posterior de la mandíbula, entre el proceso condiloideo y el proceso angular, es recto y dirigido verticalmente en MPA-84-5-I-D y MPA-85-4-I-D, y con el proceso angular sin proyectarse posteriormente, mientras que en los ciervos sudamericanos actuales el proceso angular se encuentra proyectado posteriormente en distintas proporciones, y el borde posterior de la mandíbula es cóncavo. En síntesis, de acuerdo con los rasgos determinados por autores anteriores, es posible asignar los ejemplares MPA84-5-I-D y MPA-85-4-I-D a Morenelaphus sp.

Comentarios. Todos los materiales referidos han sido hallados en capas asignadas al Piso/Edad Lujanense (Pleistoceno Tardío-Holoceno Temprano). Algunos de estos materiales fueron mencionados previamente en publicaciones (MPA-85-189-I-D; ver Tomassini et al., 2010) y acertadamente identificados como Morenelaphus sp. Los materiales procedentes de Playa del Barco han sido hallados en capas correspondientes a las facies 1 (conglomerados) y 2 (areniscas limosas) de Vega et al. (1989) asignados al Pleistoceno Tardío, según dataciones previamente obtenidas en la localidad $(16.400 \pm 320$ años AP y $12.000 \pm 110$ años
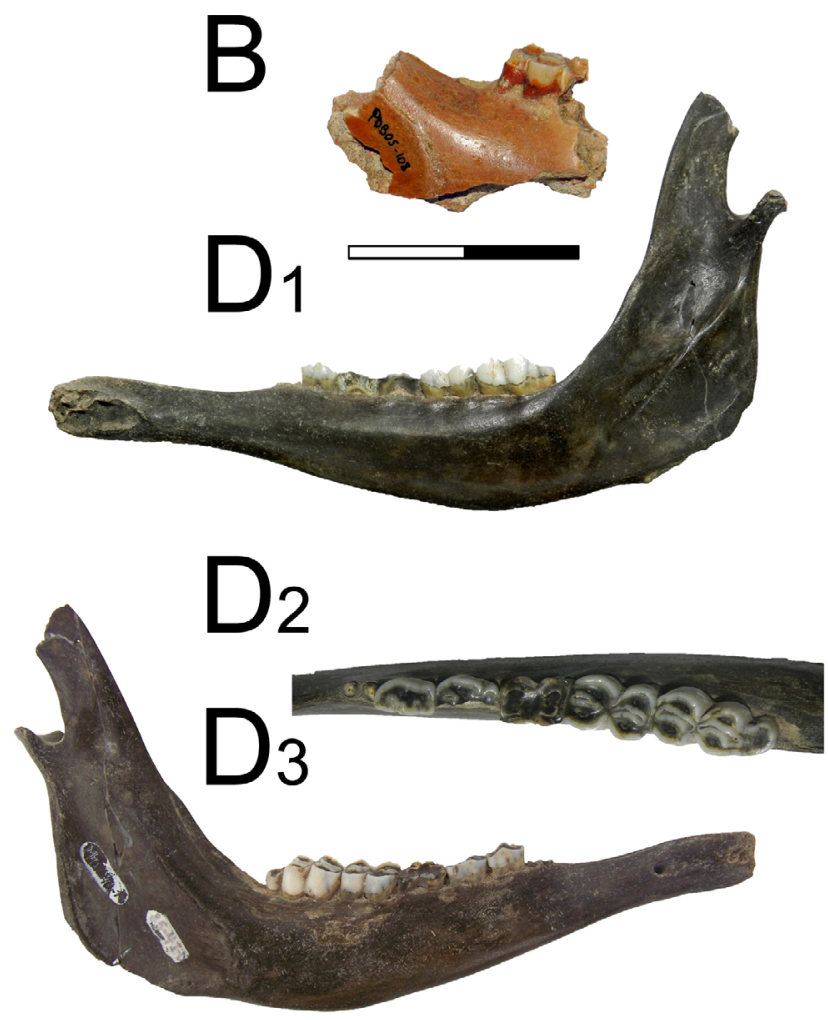

Figura 5. Restos de mandíbulas asignadas a Morenelaphus sp. A, MD-PDB-05-02 en vista lateral; B, MD-PDB-05-108 en vista lateral; C, MPA-84-5-I-D en vistas medial $\left(\mathbf{C}_{1}\right)$, oclusal $\left(\mathbf{C}_{2}\right)$ y lateral $\left(\mathbf{C}_{3}\right)$; D, MPA-85-4-I-D en vistas medial $\left(\mathbf{D}_{1}\right)$, oclusal $\left(\mathbf{D}_{2}\right)$ y lateral $\left(\mathbf{D}_{3}\right)$. Escalas $=50$ mm.

Figure 5. Mandibular remains assigned to Morenelaphus sp. A, lateral view of MD-PDB-05-02; B, lateral view of MD-PDB-05-108; C, MPA-84-5-I-D in medial $\left(\mathbf{C}_{1}\right)$, occlusal $\left(\mathbf{C}_{2}\right)$ and lateral $\left(\mathbf{C}_{3}\right)$ views; $\mathbf{D}$, MPA-85-4-I-D in medial $\left(\mathbf{D}_{1}\right)$, occlusal $\left(\mathbf{D}_{2}\right)$ and lateral $\left(\mathbf{D}_{3}\right)$ views. Scale bars $=50$ mm. 
AP; ver Aramayo, 1997; Aramayo et al., 2002; Tomassini et al., 2010). Estos niveles serían correlacionables a la Sección Media de la Secuencia Agua Blanca (sensu Deschamps, 2005).

Para esta etapa del Pleistoceno muchos autores consideraron que sólo habitaba la especie Morenelaphus lujanensis. Sin embargo, Alcaraz (2010) menciona la presencia de ambas especies de este género para el Pleistoceno Tardío de la Provincia de Buenos Aires, Argentina. De esta manera, los ejemplares aquí presentados podrían corresponder a cualquiera de las dos especies del género. La naturaleza fragmentaria de estos restos no permite una asignación más precisa.

La asignación de MD-PDB-05-110 a Morenelaphus constituye un importante hallazgo a nivel paleobiológico, ya que este ejemplar es el primer ejemplar sin astas, publicado hasta el momento para este género y para los cérvidos fósiles sudamericanos, y son escasos los trabajos donde se conocen hembras fósiles asignadas a especies que han sido caracterizadas mediante cornamentas (e.g. Eocoileus gentryorum, Sangamona fugitiva, Megaloceros giganteus; Kurtén, 1979; Webb, 2000; Vislobokova, 2013). Si bien los ejemplares machos de las especies de Cervidae se caracterizan por poseer astas, existen algunas excepciones, como Rangifer tarandus, donde ambos sexos poseen astas. En efecto, la confirmación de hembras sin astas en Morenelaphus asocia este género con los demás cérvidos, excluyéndolo de una posible filiación con Rangifer.

\section{Morenelaphus lujanensis (Ameghino, 1888) Kraglievich, 1932 \\ (Figuras 6A-C)}

Material asignado. MPA-85-107-I-D, sector posterior del cráneo con parte de los parietales, frontales y occipitales, asta derecha casi completa y asta izquierda incompleta (Figuras 6A-C); MPA-85-396-I-D, asta izquierda casi completa y parte del frontal izquierdo (Figura 6D).

Procedencia geográfica y estratigráfica. Localidad de Playa del Barco (6134'52"'O y 3900'09"S, partido de Coronel Rosales, Provincia de Buenos Aires; Figura 1C), niveles conglomerádicos asignados al Pleistoceno Tardío (Vega et al., 1989; Aramayo \& Manera de Bianco, 1989; Zavala \& Quattrocchio, 2001; Aramayo et al., 2002; Tomassini et al., 2010).

Descripción. Este material se asigna al género Morenelaphus por presentar la combinación de caracteres antes mencionados y además: 1) candil $2^{\circ}$ y $3^{\circ}$ paralelos entre sí; 2) las astas vuelven a curvarse hacia el plano sagital después del $2^{\circ}$ candil; 3) $4^{\circ}$ candil bifurcado produciendo una rama dorsal menos desarrollada que la rama posteroventral. Los ejemplares son asignados a la especie Morenelaphus lujanensis por presentar la siguiente combinación de caracteres: 1) cara anterior e interna de las astas cubiertas por una gran cantidad de tubérculos o verrugas que desaparecen en la parte superior donde son reemplazados por surcos longitudinales (Ameghino, 1888; Carette, 1922); 2) conformación de la vara con doble curvatura en "S", dirigiéndose primeramente en sentido póstero-ventral y antes de llegar al segundo candil, reorientándose en sentido anterior, donde se acentúa a nivel del tercer candil (Carette, 1922; Kraglievich, 1932; Alcaraz, 2010); 3) vara fuertemente curvada hacia atrás después del $3^{\circ}$ candil; 4) $2^{\circ}$ y $3^{\circ}$ candil dirigidos hacia arriba y divergente a la dirección de la garceta; 5) cara posterior y externa lisa o con leves surcos longitudinales (Carette, 1922).

Comentarios. Previamente, Menegaz \& Ortiz Jaureguizar (1995) y Ferrero et al. (2007) mencionan la presencia de Morenelaphus lujanensis para la localidad Playa del Barco, sin realizar una descripción y análisis detallado. A pesar de que no mencionan un número de colección, es posible que estos autores se refieran a los materiales aquí reportados. Por otro lado, no fueron incluidos materiales de esta localidad en los análisis y mapas de Alcaraz (2010).

\section{Antifer Ameghino, 1889 \\ Antifer sp. \\ (Figura 7)}

Material asignado. MPB 3947, fragmento proximal de asta izquierda de gran tamaño (Figura 7).

Procedencia geográfica y estratigráfica. Costa de la localidad de San Bernardo (Figura 1B), Partido de la Costa, Provincia de Buenos Aires, Argentina, posiblemente proveniente de la Formación Pampeano, Pleistoceno.

Descripción. Este ejemplar es asignado al género Antifer por presentar la siguiente combinación de caracteres: 1) asta con la cara interna cóncava y la cara externa convexa (Ameghino, 1888); 2) ambas caras con crestas y canales longitudinales muy marcados (Zurita \& Lutz, 2002); 3) pedúnculo circular en la base y comprimiéndose distalmente (Zurita \& Lutz, 2002; Alcaraz \& Francia, 2013). Además, en el ejemplar se puede observar la base de la garceta, que indica un ángulo recto respecto a la vara. La naturaleza fragmentaria del ejemplar no permite una asignación específica.

Comentarios. Antifer ensenadensis es considerado taxón exclusivo del Ensenadense en la Región Pampeana por Cione \& Tonni (2005); sin embargo, Labarca \& Alcaraz (2011) cita a esta especie en el Lujanense de la Formación Toropí (Provincia de Corrientes). Por otra parte, Antifer ultra Ameghino, mencionado como taxón exclusivo del Bonaerense por Cione \& Tonni (2005) ha sido registrado en la Mesopotamia (Provincia de Entre Ríos) en sedimentos asignados al Lujanense (Formación Arroyo Feliciano, ver Alcaraz et al., 2005).

Antifer sp. a su vez, se registra en la Formación Toropí en la Provincia de Corrientes (Alcaraz \& Zurita 2004), con una datación OSL referible al Lujanense (Tonni et al., 2005). Además, el género Antifer se registra en el Pleistoceno Tardío en Uruguay (Kraglievich 1932), Chile (Casamiquela, 1968, 1984; Tavera, 1978; Labarca \& López, 2006) y sur de Brasil (Souza Cunha \& Magalhaes 1981). A pesar de que MPB 3947 no presenta una asignación estratigráfica precisa, numerosos autores han planteado que Antifer no se encuentra en el Piso/ Edad Lujanense del sector bonaerense de la Región Pampeana (Alcaraz et al., 2005; Ferrero et al., 2007; Alcaraz, 2010), por lo 


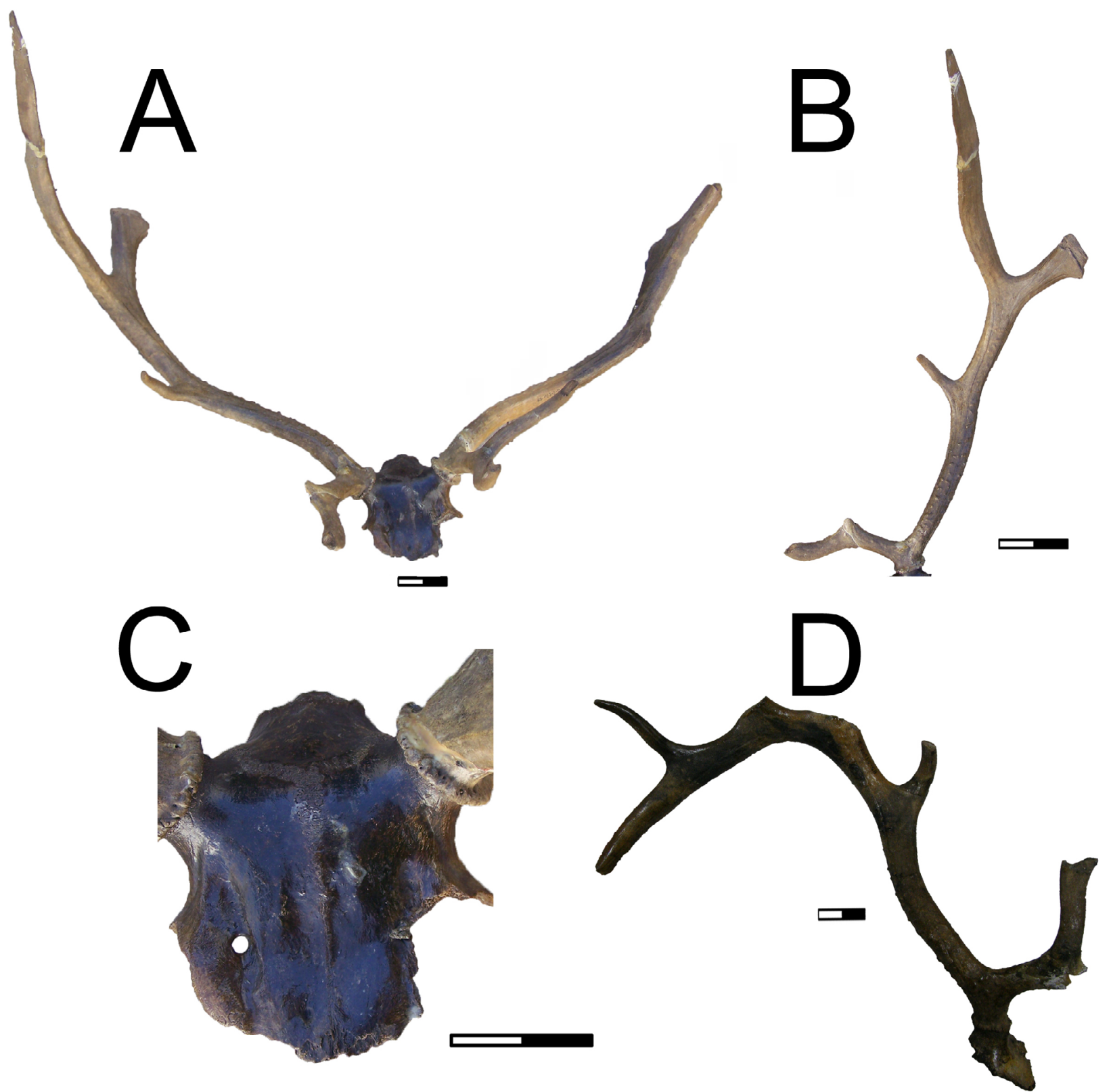

Figura 6. Restos de Morenelaphus lujanensis. A-C, MPA-85-107-I-D en vista frontal (A), asta derecha en vista medial (B) y detalle del cráneo en vista dorsal (C); D, MPA-85-396-I-D en vista medial. Escalas $=50 \mathrm{~mm}$.

Figure 6. Morenelaphus lujanensis remains. A-C, frontal view of MPA-85-107-I-D (A), right antler in medial view (B) and skull detail in dorsal view (C); D, MPA-85-396-I-D in medial view. Scale bars $=50 \mathrm{~mm}$.

cual podría corresponder al Piso/Edad Bonaerense (Pleistoceno Medio) o Ensenadense (Pleistoceno Temprano-Medio).

\section{Ozotoceros Ameghino, 1891}

Ozotoceros cf. O. bezoarticus (Linneo, 1758) (Figura 8)

Material asignado. MLS 243, parte proximal de asta derecha (Figura 8).

Procedencia geográfica y estratigráfica. Material hallado en las barrancas del Río Salado, a pocos metros del cruce de este cauce con el puente de la Ruta Nacional 7 (34³6’39.05”S y $60^{\circ} 57^{\prime} 26.28^{\prime \prime} \mathrm{O}$ ), cerca de la localidad de Junín (Figura 1A), Provincia de Buenos Aires, Argentina en capas de color verdoso, asignadas al Miembro Guerrero de la Formación Luján (Pleistoceno Tardío, Tonni et al., 2003).

Descripción. El material podría ser asignado a la especie Ozotoceros bezoarticus por presentar la siguiente combinación de caracteres: 1) asta rebifurcada, dicotómica, irregular, trirramosa (Alcaraz, 2010); 2) sección de la vara cilíndrica; 3) superficies con estriaciones; 4) garceta dirigida anterodorsalmente, simple, en ángulo obtuso con el pedúnculo y ángulo agudo con la vara, curvada dorsalmente (Ameghino, 

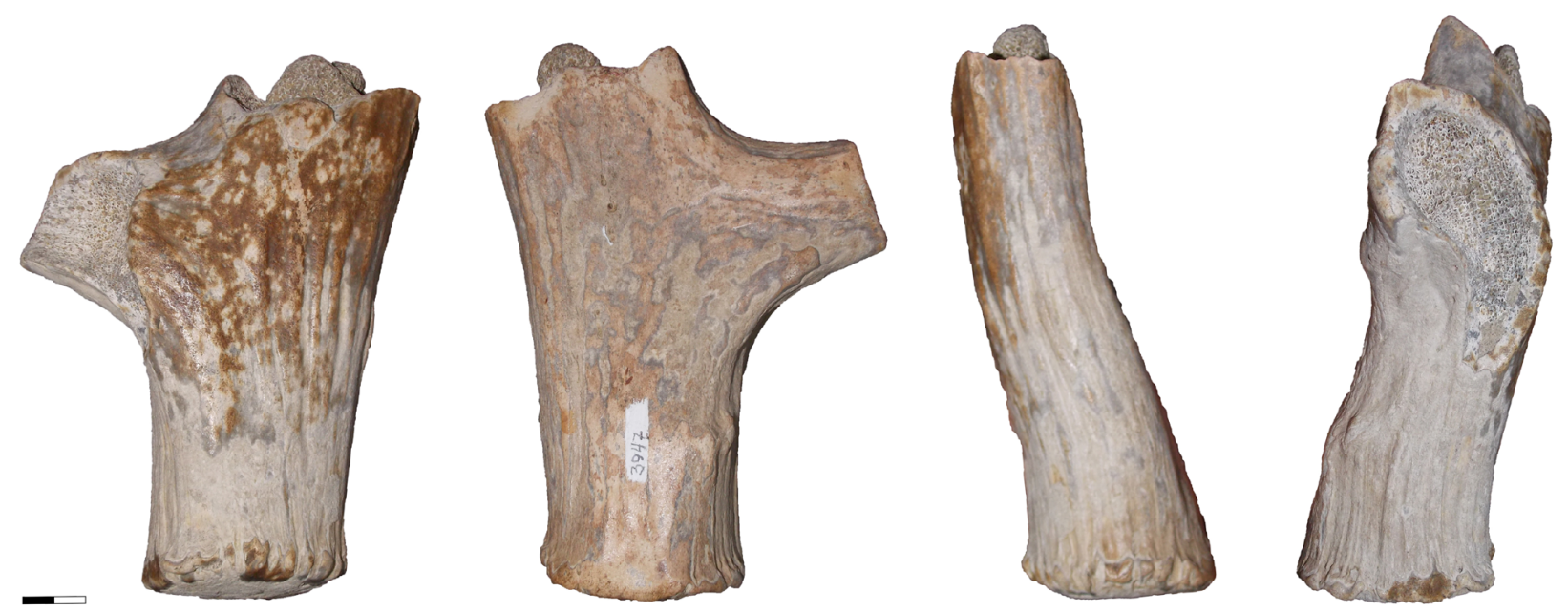

Figura 7. MPB 3947, resto de asta asignada a Antifer sp. A, vista medial; B, vista lateral; C, vista posterior; D, vista anterior. Escala $=20 \mathrm{~mm}$.

Figure 7. MPB 3947, antler remain assigned to Antifer sp. A, medial view; B, lateral view; C, posterior view; $\mathbf{D}$, anterior view. Scale bar $=20 \mathrm{~mm}$.

1891). Debido a la naturaleza fragmentaria del material consideramos como Ozotoceros cf. bezoarticus la asignación más apropiada. El asta se encuentra fragmentada en la vara después de la raíz de la garceta. La roseta presenta excrecencias óseas bien marcadas y numerosas, algunas grandes y más sobresalientes que las demás. El pedúnculo es corto y presenta leves crestas con tubérculos pequeños. La garceta es lisa.

Comentarios. Varios autores aseveran la ausencia de Ozotoceros bezoarticus en el Pleistoceno de la Región Pampeana (Menegaz, 2000; Alcaraz, 2010). Sin embargo, numerosos restos provenientes del Pleistoceno Tardío de la Provincia de Buenos Aires presentan una morfología y tamaño similar a los representantes actuales de $O$. bezoarticus (ver Prado et al., 1987; Menegaz \& Tonni, 1992; Deschamps \& Borromei, 1992), aunque otras revisiones asumen que los restos hallados no son convincentes para asignarlos a O. bezoarticus (Menegaz \& Tonni, 1985; Menegaz, 2000). Recientemente se ha reportado la presencia de esta especie para el Pleistoceno Tardío de la Provincia de Santa Fe (Vezzosi, 2010), donde previamente existían dudas sobre su presencia (Menegaz \& Ortiz Jaureguizar, 1995). Las capas verdosas aflorantes en las barrancas del Río Salado del partido de Junín corresponden al Miembro Guerrero de la Formación Luján, asignado al Pleistoceno Tardío (Tonni et al., 2003). En estas capas se han descrito numerosos materiales del Pleistoceno Tardío para localidades cercanas (Bogan et al., 2008; 2010). De esta manera, el material aquí descrito sería el primer registro pleistocénico para la Provincia de Buenos Aires.

Hippocamelus Leuckart, 1816

Hippocamelus sulcatus Ameghino, 1888 (Figura 9)

Material asignado. MLS 242, fragmento proximal de asta (Figuras 9A-B).
Material referido. MACN-A 1119, tipo de Cervus latus Ameghino, 1889 (Figura 9C).

Procedencia geográfica y estratigráfica. MLS 242 proviene de las barrancas del Río Salado, a pocos metros del cruce de este cauce con el puente de la Ruta Nacional 7 (3436'39.05'S y $60^{\circ} 57^{\prime} 26.28^{\prime \prime} \mathrm{O}$ ), cerca de la localidad de Junín (Figura 1A), Provincia de Buenos Aires, Argentina en capas de color verdoso, asignadas al Miembro Guerrero de la Formación Luján (Pleistoceno Tardío, Tonni et al., 2003). MACN-A 1119 procede del Miembro Guerrero de la Formación Luján de la localidad de Olivera, Provincia de Buenos Aires (Tonni et al., 2003).

Descripción. El material es asignado a esta especie por presentar la siguiente combinación de caracteres: 1) superficie del asta cubierta por profundos surcos longitudinales, muy marcadas sobre todo a nivel del pedúnculo (Ameghino, 1882; Alcaraz, 2010); 2) asta bifurcada desde la base sin pedúnculo o formando un corto pedúnculo (Alcaraz, 2010); 3) una rama anterior y otra posterior cilíndrico-comprimidas en sentido transversal (Alcaraz, 2010). El asta MLS 242 se encuentra muy fragmentada conservando parte de la rama posterior y una pequeña parte de la rama anterior. Por otro lado, el material tipo de "Cervus latus" (MACN-A 1119) posee los rasgos 2 y 3 mencionados arriba, por lo cual puede ser atribuido a este taxón. La ausencia de surcos profundos longitudinales muy marcados puede deberse a que el espécimen correspondería a un ejemplar joven.

Comentarios. Hippocamelus sulcatus es una especie cuyas astas son muy similares a las de los representantes actuales de este género $H$. bisulcus y $H$. antisiensis. Sin embargo, para la Región Pampeana y para la Provincia de Corrientes (y con dudas para la Provincia de Córdoba; ver Castellanos, 1944; Alcaraz, 2010) se han dado a conocer algunos materiales asignados a H. sulcatus (Ameghino, 1889; Carette, 1922; Kraglievich, 1932; Menegaz, 2000; Alcaraz, 2000; Alcaraz \& Francia, 2013). Con relación a C. latus, Menegaz (2000) lo asigna a Hippocamelus sulcatus; sin embargo, en la última revisión integral de Alcaraz (2010) este taxón es considerado 

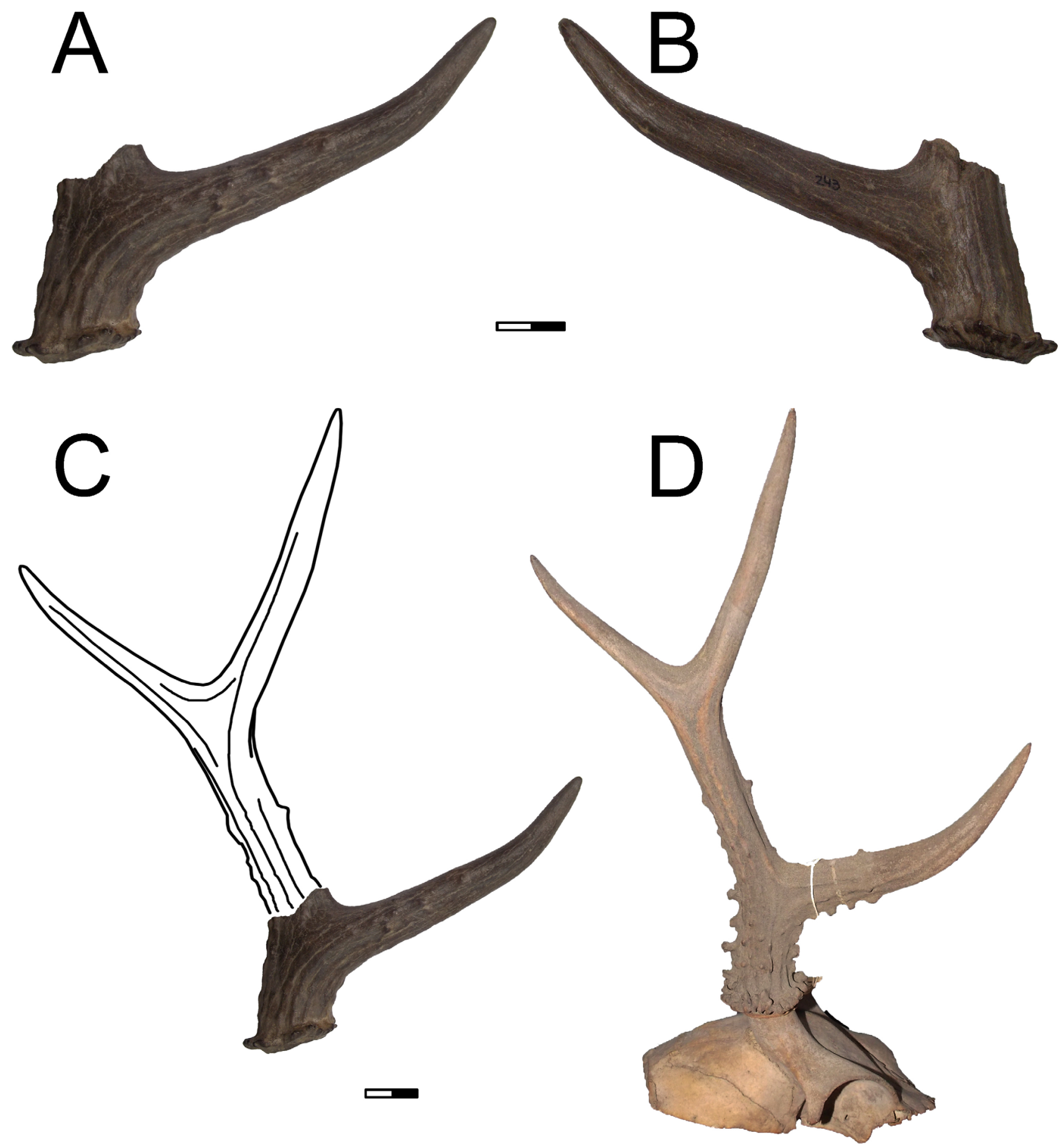

Figura 8. MLS 243, resto de asta asignada a Ozotoceros bezoarticus. A, vista lateral; B, vista medial; C-D, reconstrucción de toda la cornamenta en vista lateral (C) comparada con el ejemplar actual MACN 11.9 (D, revertido). Escalas $=20 \mathrm{~mm}$.

Figura 8. MLS 243, antler remain assigned to Ozotoceros bezoarticus. A, lateral view; B, medial view; C-D, antlers reconstruction in lateral view (C) compared with MACN 11.9 (D, reversed). Scale bars $=20 \mathrm{~mm}$. 


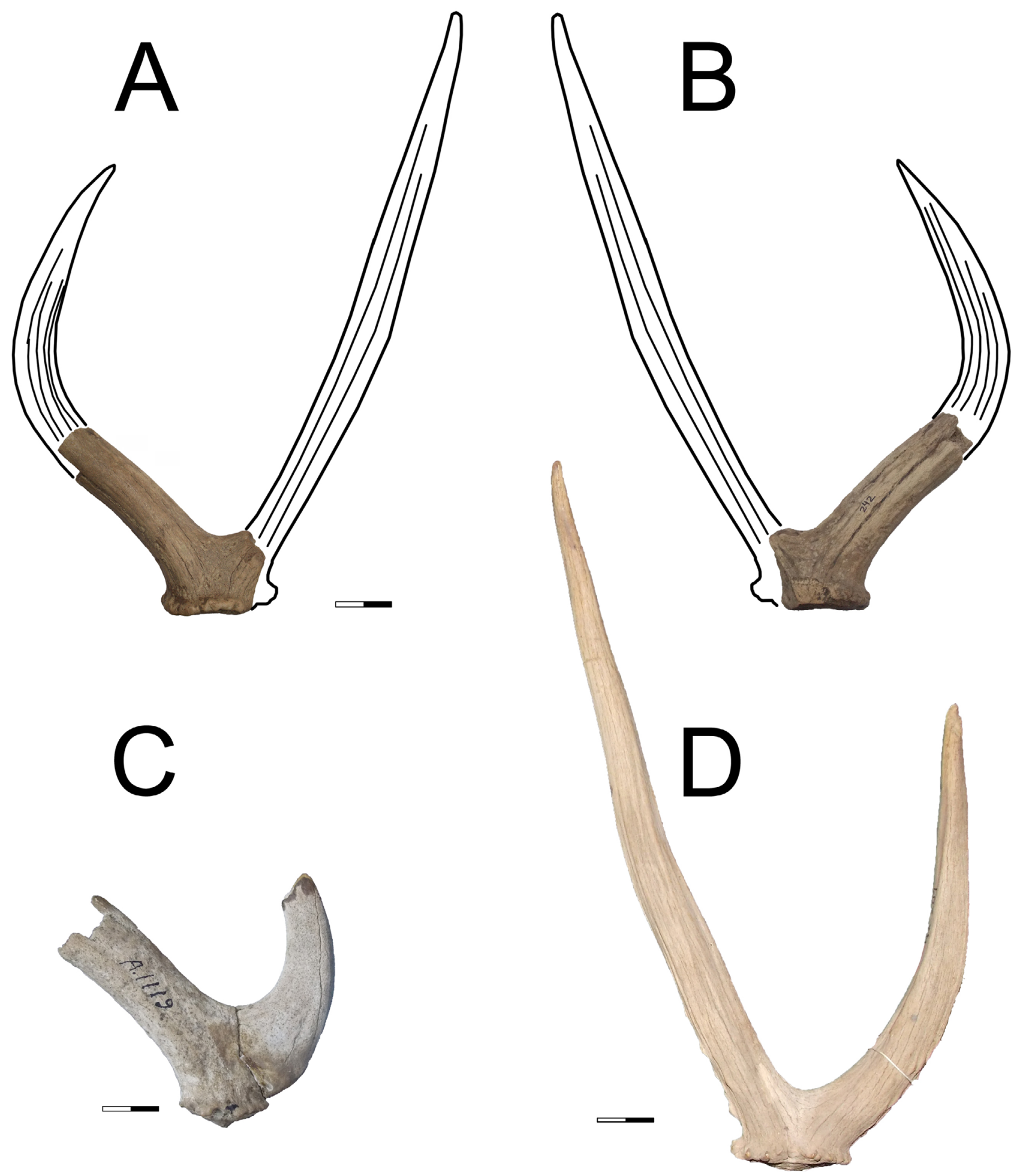

Figura 9. Restos de astas asignadas a Hippocamelus sulcatus. A-B, MLS 242 en vistas lateral y medial; C, MACN-A 1119, tipo de Cervus latus Ameghino (1889); D, ejemplar actual de H. antisiensis (MACN 42.201). Escalas $=20 \mathrm{~mm}$.

Figure 9. Remains of antlers assigned to Hippocamelus sulcatus specimens assigned. A-B, MLS 242 in lateral and medial views; C, MACN-A 1119, tipe of Cervus latus Ameghino (1889); D, current specimen of $H$. antisiensis (MACN 42.201). Scale bars $=20 \mathrm{~mm}$. 
como cf. Hippocamelus. En esta contribución, proponemos asignarlo como $H$. sulcatus, debido a la presencia de los rasgos 2 y 3 descritos más arriba.

\section{DISCUSIÓN}

El registro paleobiogeográfico del género Morenelaphus es relativamente amplio en el territorio argentino, hallándose en las provincias de Buenos Aires, Santa Fe, Entre Ríos (Figura 10A), Corrientes, Chaco y Formosa (Alvarez, 1974; Tonni \& Scillato-Yané, 1997; Scillato-Yané et al., 1998; Menegaz, 2000; Alcaraz \& Carlini, 2003; Zurita et al., 2004; Ferrero et al., 2007; Zurita et al., 2009; Alcaraz, 2010; Alcaraz \& Francia, 2013). Además, se lo ha registrado en Brasil (Souza Cunha \& Magalhães, 1981; Scherer et al., 2007; Lopes \& Buchmann, 2010; Pereira et al., 2012, entre otros), Paraguay (Carlini \& Tonni, 2000) y Uruguay (Kraglievich, 1932; Ubilla, 1996). De acuerdo a Alcaraz (2010), el registro más austral de Morenelaphus en áreas extraandinas se encuentra a las $38^{\circ} \mathrm{S}$ en el centro de la Provincia de Buenos Aires; sin embargo, no menciona ningún registro costero. Deschamps \& Borromei (1992) mencionan Morenelaphus sp. para Bajo de San José (partido de Coronel Pringles), localidad que no fue mapeada en Alcaraz (2010). Pêgo (2014) en su descripción de diversos materiales dentarios de Morenelaphus procedentes de Brasil, hace referencia a ciertos especímenes provenientes de la costa sur de la Provincia de Buenos Aires (MLP 86-VI23-81, Centinela del Mar, partido de General Alvarado; MLP 94-II-1-54, "Cascada de Palaeolama", partido de Coronel Dorrego), los cuales tampoco fueron incluidos por Alcaraz (2010). Los ejemplares MPA-85-189-I-D, MPA-85-251-I-D y MD-PDB-05-110 dados a conocer en el presente trabajo, extienden la distribución geográfica de Morenelaphus hasta los $39^{\circ} \mathrm{S}$. Sin embargo, es importante remarcar la escasez de registros en el sur de la Región Pampeana. La sistemática de los ciervos fósiles se encuentra basada en la morfología de las astas, por lo cual resulta dificultoso afinar la asignación de ciertos materiales que no presentan astas asociadas (e.g. MPA-85-189-I-D).

En el caso de Antifer sucede algo similar. A pesar del gran tamaño que presentan las astas de este género (posiblemente correspondientes a animales de mayor tamaño que los representantes del género Morenelaphus), sus registros son más escasos que los de Morenelaphus, habiéndose hallado especímenes en los Pisos/Edades Ensenadense y Bonaerense de las provincias de Buenos Aires y Santa Fe (Argentina) (Castellanos, 1945; Menegaz, 2000), y en el Piso/Edad Lujanense de las provincias de Entre Ríos y Corrientes dentro del territorio argentino, como así también en Uruguay y Brasil (Castellanos, 1924; Kraglievich, 1932; Alcaraz \& Zurita, 2004; Ubilla et al., 2004; Alcaraz et al., 2005; Ferrero et al., 2007; Ferrero \& Noriega, 2009); es importante mencionar la ausencia de registros fehacientes en la costa atlántica de la Región Pampeana. El registro de Antifer sp. en la localidad de San Bernardo (partido de la Costa, Provincia de Buenos Aires) se suma a los restos procedentes de las localidades de Mar del Sur (MLP 07-IX-1-1) y Mar del Plata (MLP 29-9-
1-1), mencionados por Alcaraz (2010) sin brindar mayores especificaciones, y que corresponderían a los restos más australes en la Provincia de Buenos Aires (Figura 10B). Lamentablemente la procedencia estratigráfica de MPB 3947 es incierta, y es probable que este especimen provenga de capas asignables al Pleistoceno Medio, ya que no existen registros concretos de Antifer en el Pleistoceno Tardío de la Provincia de Buenos Aires (Menegaz, 2000; Alcaraz, 2010).

El registro paleontológico de Hippocamelus sulcatus es aún más escaso que los taxones anteriores (Figura 10C), ya que sólo presenta unos pocos registros en el norte de la Provincia de Buenos Aires y un registro en la Mesopotamia argentina (Alcaraz, 2010; Alcaraz \& Francia, 2013), más algunos pocos en Uruguay (Kraglievich, 1932). Los registros correspondientes a la Provincia de Buenos Aires provienen de sedimentos asignados al Piso/Edad Bonaerense (Pleistoceno Medio), y el único registro hallado en la Provincia de Corrientes, corresponde al Piso/Edad Lujanense (Pleistoceno Tardío). Sin embargo, Menegaz \& Ortiz Jaureguizar (1995) asignan los materiales (MLP 9-23, tipo de "Furcifer sulcatus" Ameghino, 1889 y MLP 9-11, tipo "F. seleniticus" Ameghino, 1889) sinonimizados con $H$. sulcatus a la parte inferior del Piso/Edad Lujanense y "Cervus latus" (MACN-A 1119) a la parte superior del Lujanense (Miembro Guerrero de la Formación Luján de la localidad de Olivera). Posteriormente, Alcaraz (2010) asigna el material tipo de "Cervus latus" como cf. Hippocamelus, alegando que no presenta ningún rasgo diagnóstico. Como se demuestra en este trabajo, este último material presenta al menos dos rasgos que permiten asignarlo a $H$. sulcatus.

El registro aquí dado a conocer de Ozotoceros cf. O. bezoarticus en el Pleistoceno Tardío de Junín (Figura 10D), norte de la Provincia de Buenos Aires, representa el registro más antiguo de la especie ya que previamente se la conocía en sedimentos aflorantes en el Arroyo Napostá Grande correspondientes al Piso/Edad Platense (ver Deschamps, 2005). Sin embargo, esta especie se registra en el Pleistoceno Tardío de la Provincia de Santa Fe (Vezzosi, 2010). Es necesario realizar un análisis más profundo de los materiales hallados en la costa de la Provincia de Buenos Aires, procedentes de niveles asignados al Pleistoceno Tardío (e.g. Deschamps \& Borromei, 1992; Prado et al., 1987).

Varios autores (e.g. Lydekker, 1915; Churcher, 1966) han postulado la posible sinonimia de Morenelaphus o de Antifer, con el género Blastoceros. Sin embargo, los tres taxones se reconocen por rasgos morfológicos bien distintivos, particularmente en las bifurcaciones de las ramas, el tamaño y proporciones de las astas, y el orden en que se ubican las bifurcaciones (Cabrera, 1924; Alcaraz \& Zurita, 2004). A estas diferencias reconocidas por autores previos, se suma otra diferencia planteada en la presente contribución, que radica en los tubérculos y surcos. En Blastoceros existen profundos surcos acompañados por grandes tubérculos que se ubican en la misma orientación que los surcos (es decir, próximodistalmente ubicados), y están presentes a lo largo de toda la superficie del asta y en todo el perímetro (ver Figura 11). En Morenelaphus los tubérculos están bien desarrollados del 

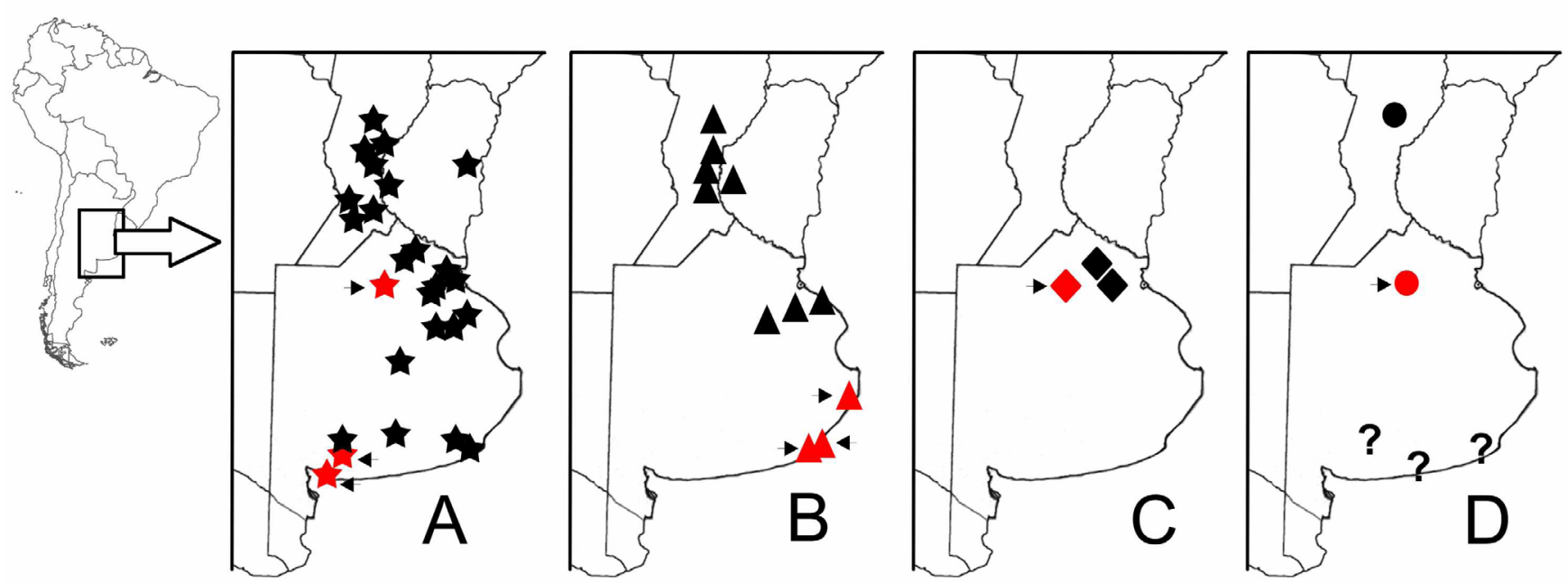

Figura 10. Registros pleistocénicos en la Región Pampeana de los taxones tratados en este trabajo. A, Morenelaphus spp.; B, Antifer spp.; C, Hippocamelus sulcatus; D, Ozotoceros bezoarticus. Los registros nuevos aportados en este trabajo están indicados com flecha.

Figure 10. Pleistocene records of the taxa mentioned in the Pampean Region. A, Morenelaphus spp.; B, Antifer spp; C, Hippocamelus sulcatus; D, Ozotoceros bezoarticus. New records of this work are indicated by arrow.

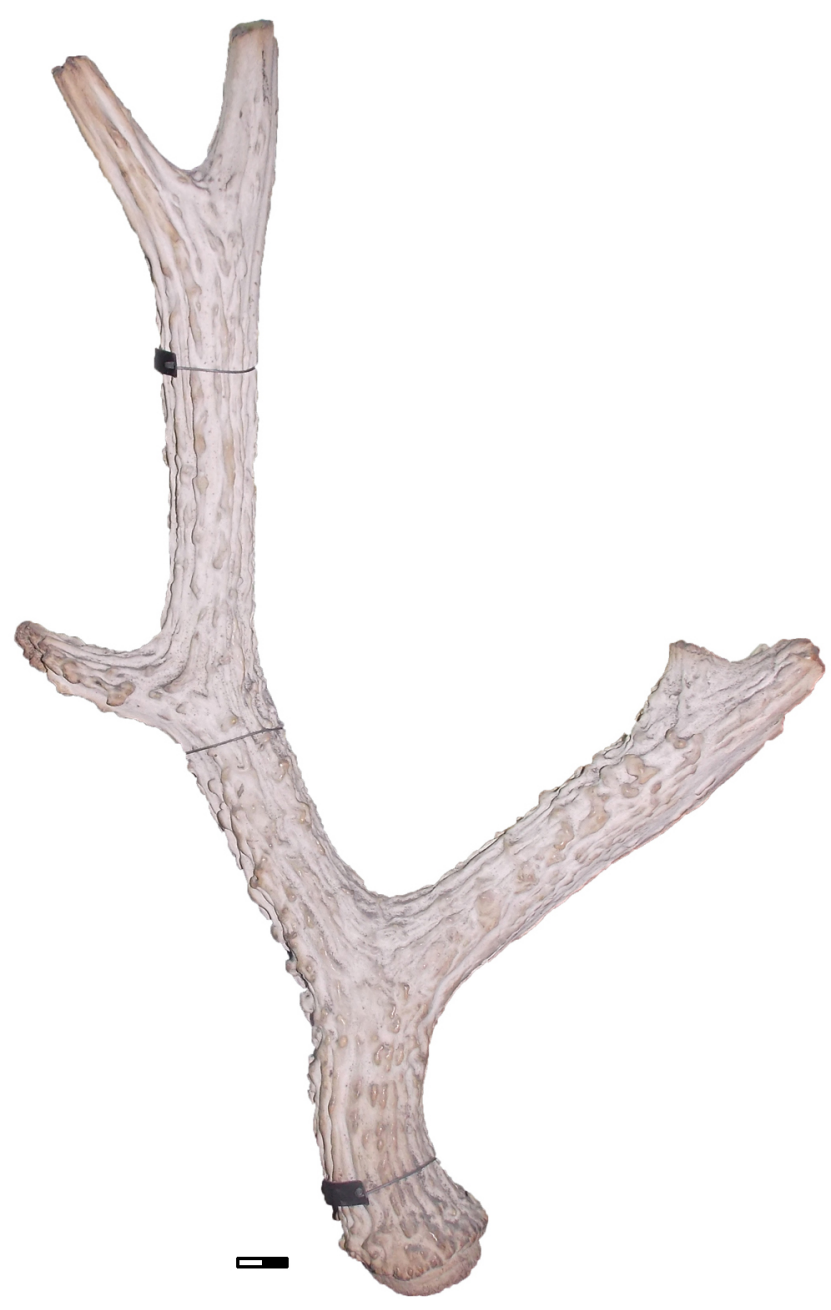

Figura 11. MACN-Pv 5127, asta asignada a Blastoceros dichotomus. Escala $=20 \mathrm{~mm}$.

Figure 11. MACN-Pv 5127, antler assigned to Blastoceros dichotomus. Scale bar $=20 \mathrm{~mm}$. lado interno y posterior del asta, de forma aleatoria, mientras que los surcos no se encuentran asociados a los tubérculos. Finalmente, en Antifer existen profundos surcos que no se encuentran asociados a tubérculos. Aunque no se han dado a conocer registros concretos de Blastoceros dichotomus para el Pleistoceno Tardío, existe un asta completa en las colecciones del Museo Argentino de Ciencias Naturales (MACN-Pv 5127, ver Figura 10), que no presenta una asignación estratigráfica precisa, pero que en la ficha del ejemplar dice "Pampeano de Canal del Norte, Buenos Aires". Canal del Norte es uno de los nombres con el que se conoce a un importante curso fluvial que une el Río Paraná de las Palmas (a la altura de Baradero) y el Río Salado (a la altura de la Laguna Mar Chiquita, partido de Junín). Este canal y sus afluentes (e.g. Río Arrecifes, Río Salto) han sido fruto de numerosos hallazgos de fauna pleistocena (Soibelzon et al., 1999; Bogan et al., 2008; 2010) por lo cual el asta de Blastoceros dichotomus podría provenir de capas asignadas al Pleistoceno Tardío del norte bonaerense. A pesar de que no existen registros estratigráficos precisos, la presencia de esta especie a fines del Pleistoceno sería concordante con los resultados obtenidos a partir de datos moleculares (ver Marquez et al., 2006). Sin embargo, varios autores han afirmado que esta especie no se registra en el Pleistoceno Tardío de la Región Pampeana (Menegaz \& Ortiz Jaureguizar, 1995; Alcaraz, 2010; Alcaraz \& Francia, 2013), por lo cual queda pendiente el hallazgo de nuevos materiales con procedencia estratigráfica precisa que puedan dar luz sobre esta problemática.

De confirmarse la presencia del ciervo de los pantanos en el Pleistoceno Tardío, la diversidad de Cervidae en la Provincia de Buenos Aires en esta etapa, estaría dada por la presencia de cuatro cérvidos grandes, Morenelaphus lujanensis, $M$. brachyceros, Epieuryceros truncus y Blastocerus dichotomus, y dos especies de mediano tamaño, Ozotoceros bezoarticus e Hippocamelus sulcatus. Esta abundancia de taxones de la misma familia es similar a la señalada para la Región 
Mesopotámica argentina, con la diferencia de que en la misma habitaba Antifer ultra en tiempos del Pleistoceno Tardío y no hay evidencia de la presencia de E. truncus, una especie hasta ahora restringida sólo a la Provincia de Buenos Aires (Alcaraz et al., 2005; Ferrero et al., 2007; Alcaraz, 2010; Alcaraz \& Francia, 2013). Esta gran diversidad de Cervidae podría deberse a la plasticidad del grupo para adaptarse a condiciones ambientales variables (Alcaraz \& Francia, 2013), algo que podría correlacionarse también con el hecho de su amplia distribución geográfica. Sin embargo, Menegaz \& Ortiz Jaureguizar (1995) proponen que los ciervos grandes como Antifer tenían preferencias por ambientes abiertos con vegetación de tipo arbustiva y clima árido. Por otro lado, se observa una mayor abundancia de restos fósiles de Morenelaphus sobre los demás taxones del Piso/ Edad Lujanense de la Región Pampeana. Esto podría estar indicando una dominancia de este taxón sobre los demás ciervos (e.g. H. sulcatus, E. truncus, etc.). Alcaraz (2010) menciona que Epieuryceros y Antifer comienzan a registrarse en el Piso/Edad Ensenadense de la Región Pampeana, donde no hay registros de Morenelaphus. Ante la aparición de Morenelaphus en el Piso/Edad Bonaerense (Pleistoceno Medio), una posible competencia con los taxones anteriores podría haber provocado el desplazamiento de Epieuryceros hacia el sur y Antifer hacia el norte, quedando Epieuryceros relegado al sur de la Provincia de Buenos Aires, y Antifer sobreviviendo en la Mesopotamia hasta el Pleistoceno Tardío. Por otro lado, H. sulcatus no habría sido una especie abundante, ya que sus registros no abundan en los niveles donde se lo encuentra, asignados al Piso/Edad Bonaerense (Pleistoceno Medio) y Lujanense (Pleistoceno Tardío). Lo mismo parece haber ocurrido con Paraceros fragilis, que sólo se registra en el Piso/Edad Bonaerense de la Provincia de Buenos Aires. Es posible, que Blastoceros dichotomus y Ozotoceros bezoarticus hayan estado presentes en el Piso/ Edad Lujanense, pero en forma escasa. Estos últimos taxones podrían haberse encontrado con numerosos nichos vacantes luego de la extinción de Morenelaphus, Epieuryceros, Antifer e Hippocamelus sulcatus a fines del Pleistoceno Tardío, como lo evidencian la gran cantidad de restos de $O$. bezoarticus y B. dichotomus, hallados en sitios arqueológicos del Holoceno de la Región Pampeana (Politis \& Tonni, 1982; Salemme \& Tonni, 1993; Deschamps \& Tonni, 1992; Menegaz, 2000; Politis et al., 2004).

La sistemática de los taxones fósiles de esta familia en América del Sur presenta profundos problemas, sobre todo porque la diferenciación entre taxones se ha dado exclusivamente por rasgos de las cornamentas, que son estructuras muy variables a nivel intraespecífico (ver Ameghino, 1889; Lydekker, 1893; Carette, 1922; Castellanos, 1924; Kraglievich, 1932; Churcher, 1966; Bagnalasta, 1980). Un primer adelanto a un consenso sistemático ha sido realizado por Pêgo (2014), quien a través de la morfología dentaria concluyó que no existen evidencias concretas para separar ambas especies de Morenelaphus. Futuros hallazgos con procedencia precisa, de materiales más completos y asociados a restos postcraneanos, permitirán dar luz sobre los profundos problemas sistemáticos que presentan los ciervos fósiles argentinos y precisar mejor las distribuciones geográficas y estratigráficas de los taxones.

\section{AGRADECIMIENTOS}

En primer lugar, deseamos agradecer a aquellas personas que colaboraron con las colecciones del Museo Legado del Salado (Junín), en especial a H.G. Paduani y C. Arranz, por los materiales descubiertos en las barrancas del Río Salado. Por otro lado, agradecemos a C. Oliva y R. Tomassini por facilitar materiales y fotografías. Deseamos expresar un cordial agradecimientos a los curadores de las colecciones consultadas, especialmente a S. Lucero y P. Teta (Sección Mastozoología, Museo Argentino de Ciencias Naturales "Bernardino Rivadavia"), A. Kramarz (Sección Paleontología Vertebrados, Museo Argentino de Ciencias Naturales "Bernardino Rivadavia"), M. Reguero y M. de los Reyes (Colección Paleontología Vertebrados, Museo de La Plata), Silvia Cornero, L. Rangone y F. Solomita (Museo Universitario Florentino y Carlos Ameghino, Universidad Nacional de Rosario, Rosario, Argentina; Sergio Bogan (Fundación de Historia Natural "Félix de Azara", Ciudad Autónoma de Buenos Aires, Argentina), A. Méndez (Museo Paleontológico Bariloche, Bariloche, Argentina), T.M. de Bianco y S. Aramayo (Paleontología Vertebrados, Universidad Nacional del Sur, Bahía Blanca, Buenos Aires, Argentina). Un último agradecimiento para G. Gasparini y un revisor anónimo por sus sugerencias que aportaron mucho a la calidad de este trabajo.

\section{REFERENCIAS}

Alcaraz, M.A. 2010. Sistemática de los cérvidos (Mammalia, Artiodactyla) del Pleistoceno de las áreas extraandinas de Argentina. Universidad Nacional de La Plata, Tesis Doctoral, $317 \mathrm{p}$.

Alcaraz, M.A. \& Carlini, A.A. 2003. Los cérvidos (Mammalia, Artiodactyla) de las Formaciones Toropi y Yupoí (Pleistoceno medio-tardio) de la provincia de Corrientes, Argentina. Corrientes, Comunicaciones Científicas y Tecnológicas de la Universidad Nacional del Nordeste. Available at http//www. unne.edu.ar; accessed on 04/27/2019.

Alcaraz, M.A.; Ferrero, S.B. \& Noriega, J.I. 2005. Primer registro de Antifer ultra Ameghino, 1889 (Artiodactyla: Cervidae) en el Pleistoceno de Entre Ríos. Tucumán, Temas de Biodiversidad del Litoral Fluvial Argentino II, INSUGEO, p. 65-70 (Miscelánea 14).

Alcaraz, M.A. \& Francia, A. 2013. Diversidad de Cervidae (Mammalia, Artiodactyla) en el Pleistoceno de la Provincia de Corrientes, Argentina. Revista Brasileira de Paleontologia, 16:157-166. doi:10.4072/rbp.2013.1.12

Alcaraz, M.A. \& Zurita, A.E. 2004. Nuevos registros de cérvidos poco conocidos: Epieuryceros cf. proximus Castellanos y Antifer sp. (Mammalia, Artiodactyla, Cervidae). Revista del Museo Argentino de Ciencias Naturales, 6:41-48. 
Álvarez, B.B. 1974. Los Mamíferos fósiles del Cuaternario de Bella Vista, Corrientes (Argentina). Ameghiniana, 11:295-311.

Ameghino, F. 1882. Catálogo de las colecciones de Antropología prehistórica y paleontología de Florentino Ameghino, Partido de Mercedes. Buenos Aires, Catálogo de la Sección de la Provincia de Buenos Aires (República Argentina), Exposición Continental Sudamericana, p. 35-42.

Ameghino, F. 1888. Rápidas diagnosis de algunos mamíferos fósiles nuevos de la República Argentina. Obras Completas, 5:471-480.

Ameghino, F. 1889. Contribución al conocimiento de los mamíferos fósiles de la República Argentina. Actas de la Academia Nacional de Ciencias de Córdoba, 6:1-1027.

Ameghino, F. 1902. Nota sobre algunos mamíferos fósiles nuevos o poco conocidos del Valle de Tarija. Anales del Museo Nacional de Buenos Aires, 8:1-250.

Aramayo, S.A. \& Manera de Bianco, T. 1987. Hallazgo de una icnofauna contiental (Pleistoceno tardío) en la localidad de Pehuen Có, Provincia de Buenos Aires, Argentina. Parte I: Edentata, Litopterna, Proboscidea. Parte II: Carnivora, Artiodactyla y Aves. In: CONGRESO LATINOAMERICANO DE GEOLOGÍA, 4, 1987. Actas, Santa Cruz de la Sierra, p. 516-547.

Aramayo, S. \& Manera de Bianco, T. 1989. Nuevos hallazgos de mamíferos pleistocénicos en el yacimiento de Playa del Barco, provincia de Buenos Aires. In: JORNADAS GEOLÓGICAS BONAERENSES, 1, 1985. Actas, Tandil, p. 701-712.

Aramayo, S.A. \& Manera de Bianco, T. 1996. Edad y nuevos hallazgos de icnitas de mamíferos y aves en el yacimiento paleoicnológico de Pehuen Có (Pleistoceno tardio), provincia de Buenos Aires, Argentina. Buenos Aires, Asociación Paleontológica Argentina, p. 47-57 (Publicación Especial 4).

Aramayo, S.A.; Schillizzi, R.A. \& Gutiérrez Téllez, B.M. 2002. Evolución paleoambiental del Cuaternario en la costa atlántica del sur de la provincia de Buenos Aires, Argentina. Revista de la Sociedad Geológica Española, 15:95-104.

Azorit, C.; Mohamed, A.; Carrasco, R.; Carrasco, A. \& Muñozcobo, A. 2002. Astas, esqueleto y edad del ciervo (Cervus elaphus hispanicus) de Sierra Morena oriental: Estudio de correlación. Anales de Biología, 24:195-200.

Bagnalasta, M.B. 1980. Algunas consideraciones sobre la validez del género Epieuryceros Ameghino, 1889. In: CONGRESO ARGENTINO DE PALEONTOLOGÍA Y CONGRESO LATINOAMERICANO, 2, 1978. Libro de Actas, Buenos Aires, p. 183-192.

Bogan, S.; Agnolin, F.L. \& Ramírez, J.L. 2010. Ictiofauna y herpetofauna del Pleistoceno superior continental de la localidad de Salto, provincia de Buenos Aires, Argentina. Studia Geologica Salmanticensia, 46:83-97.

Bogan, S.; De los Reyes, M.L.; Toledo, M.J. \& Ramírez, J.L. 2008. Registros fósiles de "viejas del agua" (Teleostei: Siluriformes) del Pleistoceno superior de la localidad de Salto, Buenos Aires, Argentina. Studia Geologica Salmanticensia, 44:203-212.

Cabrera A. 1929. Sobre los ciervos fósiles sudamericanos llamados Paraceros $y$ Morenelaphus. Madrid, Real Sociedad Española de Historia Natural, 55 p. (Memorias 15).

Cabrera, A. 1943. Sobre la sistemática del venado y su variación individual y geográfica. Revista del Museo de La Plata, Zoologia, 3:5-41.

Carette, E. 1922. Cérvidos actuales y fósiles de Sudamérica. Revisión de las formas extinguida pampeanas. Revista del Museo de la Plata, 26:393-472.
Carlini, A.A. \& Tonni, E.P. 2000. Mamíferos fósiles del Paraguay. Buenos Aires, Cooperación Técnica Paraguayo-Alemana, 108 p.

Casamiquela, R. 1968. Catalogación crítica de algunos vertebrados fósiles chilenos. I. Los Ciervos. La presencia de Antifer (=Blastocerus?) en el Pleistoceno Superior. Revista Universitaria, 53:101-106.

Casamiquela, R. 1984. Critical catalogue of some Chilean fossil vertebrates. I. The deers: complementary considerations on Antifer (Antifer niemeyeri n. sp.), the Pleistocene giant deer. Quaternary of South America and Antarctic Peninsula, 2:41-50.

Castellanos, A. 1924. Ciervos vivientes y extinguidos de la República Argentina (Nota preliminar). Revista de la Universidad de Córdoba, 11:111-134.

Castellanos, A. 1944. Paleontología estratigráfica de los sedimentos neógenos de la provincia de Córdoba. Rosario, Instituto de Fisiografía y Geología de la Facultad de Ciencias matemáticas, Físico-Química y Naturales aplicadas a la industria de la Universidad del Litoral, $47 \mathrm{p}$.

Castellanos, A. 1945. Nota sobre algunas cornamentas de ciervos de los géneros Epieuryceros, Antifer y Paraceros y descripción de la de Epieuryceros proximus $n$. $s p$. Rosario, Instituto de Fisiografía y Geología de la facultad de Ciencias Físico- Químicas y Naturales aplicadas a la Industria de la Universidad Nacional del Litoral, p. 5-23.

Cione, A.L.; Gasparini, G.M.; Soibelzon, E.; Soibelzon, L.H. \& Tonni, E.P. 2015. The Great American Biotic Interchange: a South American perspective. Dordrecht, Springer, 97 p. doi:10.1007/978-94-017-9792-4

Cione, A.L. \& Tonni, E.P. 1999. Biostratigraphy and chronological scale of uppermost Cenozoic in the Pampean Area, Argentina. Quaternary of South American and Antarctic Peninsula, 12:23-51.

Cione, A.L. \& Tonni, E.P. 2005. Bioestratigrafía basada en mamíferos del Cenozoico superior de la provincia de Buenos Aires, Argentina. Geología y recursos minerales de la provincia de Buenos Aires, 11:183-200.

Deschamps, C.M. 2005. Late Cenozoic mammal biochronostratigraphy in southwestern Buenos Aires province, Argentina. Ameghiniana, 42:733-750.

Deschamps, C.M. \& Borromei, A.M. 1992. La fauna de vertebrados pleistocénicos de Bajo San José (provincia de Buenos Aires, Argentina). Aspectos paleoambientales. Ameghiniana, 19:177-183.

Deschamps, C.M. \& Tonni, E.P. 1992. Los vertebrados del Pleistoceno tardío-Holoceno del arroyo Napostá Grande, provincia de Buenos Aires. Aspectos paleoambientales. Ameghiniana, 29:201-210.

Ferrero, B.S.; Brandoni, D.; Noriega, J.I. \& Carlini, A.A. 2007. Mamíferos de la Formación El Palmar (Pleistoceno tardío) de la provincia de Entre Ríos, Argentina. Revista del Museo Argentino de Ciencias Naturales, 9:109-117.

Ferrero, B.S. \& Noriega, J.I. 2009. La paleontología de vertebrados en el Cuaternario de la provincia de Entre Ríos (Argentina): estado actual y perspectivas. In: A.M. Ribeiro; S.G. Bauermann \& C.S. Scherer (eds.) Quaternário do Rio Grande do SulIntegrando Conhecimentos, Porto Alegre, Sociedade Brasileira de Paleontologia, p. 205-215 (Monografias 1).

Hershkovitz, P. 1982. Neotropical deer (Cervidae) Part I. Pudus, genus Pudu Gray. Fieldiana Zoology, 11:1-86. 
Kraglievich, J.L. 1932. Contribución al conocimiento de los ciervos fósiles del Uruguay. Anales del Museo de Historia Natural de Montevideo, 2:355-438.

Kurten, B. 1979. The stilt-legged deer Sangamona of the North-American Pleistocene. Boreas, 8:313-321. doi:10.1111/j.1502-3885.1979.tb00815.x

Labarca, R. \& Alcaraz, M.A. 2011. Presencia de Antifer ultra Ameghino (= Antifer niemeyeri Casamiquela) (Artiodactyla, Cervidae) en el Pleistoceno tardío-Holoceno temprano de Chile central (30-35 S). Andean geology, 38:156-170.

Labarca, R. \& López, P. 2006. Los mamíferos finipleistocénicos de la Formación Quebrada Quereo: (IV Región-Chile): biogeografía, bioestratigrafía e inferencias paleoambientales. Mastozoología Neotropical, 13:89-101.

Leuckart, F.S. 1816. Dissertatiuncula inauguralis de Equo bisulco Molinre, Facultatis Medicae Gottingensis con sensu pro obtinendis doctoris medicinae honoribus scripsit. Gottingen, Georg-August-Universitat.

Lopes, R.P. \& Buchmann, F.S.C. 2010. Pleistocene mammals from the southern Brazilian continental shelf. Journal of South American Earth Sciences, 31:17-27. doi:10.1016/j. jsames.2010.11.003

Loponte, D.M. 2005. Atlas osteológico de Blastocerus dichotomus (ciervo de los pantanos). Buenos Aires, Ed. Los Argonautas, 78 p.

Lydekker, R. 1893. Estudios sobre los Ungulados extinguidos de la Argentina. Paleontología Argentina. Anales del Museo de La Plata, 2:1-91.

Lydekker, R. 1915. Catalogue of the ungulate mammals in the British Museum. Vol. IV. Artiodactyla, families Cervidae (deer), Tragulidae (chevrotains), Camelidae (camels and llamas), Suidae (pigs and peccaries), and Hippopotamidae (hippopotamuses). London, British Museum, 438 p. (Rustees 4).

Marquez, A.; Maldonado, J.E.; González, S.; Beccaceci, M.D.; Garcia, J.E. \& Duarte, J.M.B. 2006. Phylogeography and Pleistocene demographic history of the endangered marsh deer (Blastocerus dichotomus) from the Río de la Plata Basin. Conservation Genetics, 7:563-575. doi:10.1007/s10592-0059067-8

Menegaz, A.N. 2000. Los Camélidos y Cérvidos del Cuaternario del Sector Bonaerense de la Región Pampeana. Universidad Nacional de La Plata, Tesis Doctoral, 206 p.

Menegaz, A.N. \& Ortiz-Jaureguizar, E. 1995. Los Artiodáctilos. In: M.T. Alberdi; G. Leone \& E.P. Tonni (eds.) Evolución biológica y climática de la región Pampeana durante los últimos cinco millones de años. Un ensayo de correlación con el Mediterráneo occidental, Madrid, Museo Nacional de Ciencias Naturales, Consejo Superior de Investigaciones Científicas, p. 311-337 (Monografías 12).

Menegáz, A.N. \& Tonni, E.P. 1985. Ozotoceros bezoarticus (Mammalia, Cervidae) in the Quaternary of Buenos Aires province, Argentina. Quaternary of South America and Antartic Peninsula, 3:43-53.

Pardiñas, U.F.; Gelfo, J.N.; San Cristóbal, J.; Cione, A.L. \& Tonni, E.P. 1996. Una tafocenosis de organismos marinos y continentales en el Pleistoceno superior en el sur de la provincia de Buenos Aires. In: CONGRESO GEOLÓGICO ARGENTINO, 13, 1996. Actas, Buenos Aires, p. 95-111.

Pêgo, F.B. 2014. Revisão taxonômica de Morenelaphus Carette, 1922 (Cetartiodactyla, Cervidae): uma abordagem morfológica dentária. Universidade Federal do Estado do Rio de Janeiro, Dissertação de Mestrado, 74 p.
Pereira, J.C.; Lopes, R.P. \& Kerber, L. 2012. New remains of late pleistocene mammals from the Chuí Creek, Southern Brazil. Revista Brasileira de Paleontologia, 15:228-239. doi:10.4072/ rbp.2012.2.10

Politis, G.; Messineo, P.G. \& Kaufmann, C.A. 2004. El poblamiento temprano de las llanuras pampeanas de Argentina y Uruguay. Complutum, 15:207-224.

Politis, G. \& Tonni, E. 1982. Arqueología de la Región Pampeana: el Sitio 2 de zanjón Seco (Pdo. de Necochea, Provincia de Buenos Aires, Argentina). Revista de Prehistoria, 3:107-140.

Prado, J.L.; Menegaz, A.N.; Tonni, E.P. \& Salemme, M.C. 1987. Los mamíferos de la fauna local Paso Otero (Pleistoceno tardío), Provincia de Buenos Aires. aspectos paleoambientales y bioestratigráficos. Ameghiniana, 24:217-233.

Salemme, M.C. \& Tonni, E.P. 1983. Paleoentozoología de un sitio arqueológico en la Pampa Ondulada: sitio Río Luján (Partido de Campana: provincia de Buenos Aires). Relaciones de la Sociedad Argentina de Antropología, 15:77-90.

Scherer, C.S.; Pitana, V.G. \& Ribeiro, A.M. 2007. Novas considerações sobre os Cervidae (Mammalia, Artiodactyla) no Quaternário do Rio Grande do Sul, Brasil. Ameghiniana, 43:39-40.

Scillato-Yané, G.J.; Tonni, E.P.; Carlini, A.A. \& Noriega, J.I. 1998. Nuevos Hallazgos de Mamíferos del Cuaternario en el Arroyo Toropí, Corrientes, Argentina. Aspectos bioestratigráficos, paleoambientales y paleozoogeograficos. In: CONGRESO LATINOAMERICANO DE GEOLOGÍA, 10, 1998. Actas, Buenos Aires, p. 263-268.

Soibelzon, L.H.; Gelfo, J.N. \& Pasquali, R.C. 1999. Bioestratigrafía del Pleistoceno en el río Arrecifes, Buenos Aires. In: CONGRESO GEOLÓGICO ARGENTINO, 14, 1999. Actas, Salta, p. 381384.

Souza Cunha, F.L.S. \& Magalhães, R.M.M. 1981. Cervídeos pleistocênicos de Santa Vitória do Palmar, Rio Grande do Sul, Brasil. In: CONGRESSO LATINO-AMERICANO DE PALEONTOLOGIA, 2, 1981. Anais, Porto Alegre, p. 795-803.

Tavera, J. 1978. Noticia sobre el hallazgo de material de cornamenta de cérvido en depósito cuaternario (Pleistoceno) de Pudahuel. Santiago, Departamento de Geología, Facultad de Ciencias Físicas y Matemáticas, Universidad de Chile, p. 26-29 (Comunicaciones 23).

Tomassini, R.L.; Montalvo, C.I.; Manera, T. \& Oliva, C. 2010. Estudio tafonómico de los mamíferos pleistocenos del yacimiento de Playa del Barco (Pehuen Co), provincia de Buenos Aires, Argentina. Ameghiniana, 47:137-152. doi:10.5710/ AMGH.v47i2.5

Tonni, E.P.; Carlini, A.A.; Zurita, A.; Frechen, M.; Gasparini, G.; Budziak, D. \& Kruck, W. 2005. Cronología y Bioestratigrafía de las unidades del Pleistoceno aflorantes en el arroyo Toropí, provincia de Corrientes, Argentina. In: CONGRESSO LATINOAMERICANO DE PALEONTOLOGÍA, 4, 2005. Resúmenes, Rio de Janeiro.

Tonni, E.P.; Huarte, R.A.; Carbonari, J.E. \& Figini, A.J. 2003. New radiocarbon chronology for the Guerrero Member of the Luján Formation (Buenos Aires, Argentina): palaeoclimatic significance. Quaternary International, 109-110:45-48. doi:10.1016/S1040-6182(02)00201-X

Tonni, E.P. \& Scillato-Yané, G.J. 1997. Una nueva localidad con mamíferos pleistocenos en el Norte de la Argentina. Aspectos paleozoogeográficos. In: CONGRESSO DA ASSOCIAÇÃO BRASILEIRA DE ESTUDOS DO QUATERNÁRIO, 6, 1997. Livro de resumos, Curitiba, ABEQUA, p. 345-348. 
Ubilla, M. 1996. Paleozoología del Cuaternario continental de la cuenca norte del Uruguay: biegeografía, cronología y aspectos climáticos-ambientales. Universidad de la Republica, Tesis doctoral, $132 \mathrm{p}$.

Ubilla, M.; Perea, D.; Aguilar, C.G. \& Lorenzo, N. 2004. Late Pleistocene vertebrates from northern Uruguay: tools for biostratigraphic, climatic and environmental reconstruction. Quaternary International, 114:129-142. doi:10.1016/S10406182(03)00048-X

Vega, V.; Rodríguez, S. \& Valente, M. 1989. Shallow marine and fluvial enviroments of Quaternary deposits in Pehuén Co Beach, Buenos Aires, Argentina. Quaternary of South America and Antarctic Peninsula, 7:51-80.

Vezzosi, R.I. 2011. Primer registro de Ozotoceros bezoarticus (Linnaeus, 1758) (Artiodactyla: Cervidae) en el Pleistoceno del centro de la provincia de Santa Fe, Argentina. In: J. Calvo; J. Porfiri; B. González Riga \& D. Dos Santos (eds) Paleontología y dinosaurios desde América Latina, Universidad Nacional de Cuyo, p. 235-244.

Vislobokova, I.A. 2013. Morphology, taxonomy, and phylogeny of megacerines (Megacerini, Cervidae, Artiodactyla). Paleontological Journal, 47:833-950. doi:10.1134/ s0031030113080017

Webb, S.D. 2000. Evolutionary history of new world Cervidae. In: E.S. Vrba \& G.B. Schaller (eds.) Antelopes, deer, and relatives: fossil record, behavioral ecology, systematics, and conservation, Yale University Press, p. 38-64.
Zavala, C.A. \& Quattrocchio, M.E. 2001. Estratigrafía y evolución geológica del río Sauce Grande (Cuaternario), provincia de Buenos Aires. Revista de la Asociación Geológica Argentina, 56:25-37.

Zurita, A.E.; Carlini, A.A.; Scillato-Yané, G.J. \& Tonni, E.P. 2004. Mamíferos extintos del Cuaternario de la provincia del Chaco (Argentina) y su relación con aquellos del este de la región pampeana y de Chile. Revista Geológica de Chile, 31:65-89.

Zurita, A.E. \& Lutz, A.E. 2002. La Fauna Pleistocena de la Formación Toropí en la Provincia de Corrientes (Argentina). Mastozoología Neotropical, 9:47-56.

Zurita, A.E.; Miño-Boilini, A.R.; Carlini, A.A.; Iriondo, M.H. \& Alcaraz, M.A. 2009. Paleontología del Chaco Oriental. Una nueva localidad con mamíferos fósiles pleistocenos en el río Bermejo (Formosa, Argentina). Revista Mexicana de Ciencias Geológicas, 26:277-288.

Received in 04 August, 2018; accepted in 02 April, 2019. 